\title{
Precision non-implantable neuromodulation therapies: a perspective for the depressed brain
}

Lucas Borrione, ${ }^{1}$ iD Helena Bellini, ${ }^{1}$ Lais Boralli Razza, ${ }^{1}$ Ana G. Avila, ${ }^{2}$ Chris Baeken, ${ }^{3,4,5,6}$ Anna-Katharine Brem, ${ }^{7,8}$ Geraldo Busatto, ${ }^{9}$ Andre F. Carvalho, ${ }^{10,11}$ Adam Chekroud, ${ }^{12,13}$ Zafiris J. Daskalakis, ${ }^{10,11}$ Zhi-De Deng, ${ }^{14,15}$ Jonathan Downar, ${ }^{16,17}$ Wagner Gattaz, ${ }^{18,19}$ Colleen Loo, ${ }^{20}$ Paulo A. Lotufo, ${ }^{21}$ Maria da Graça M. Martin, ${ }^{22}$ Shawn M. McClintock, ${ }^{23}$ Jacinta O'Shea, ${ }^{24}$ Frank Padberg, ${ }^{25}$ Ives C. Passos, ${ }^{26}$ Giovanni A. Salum, ${ }^{27}$ Marie-Anne Vanderhasselt, ${ }^{3,5,28}$

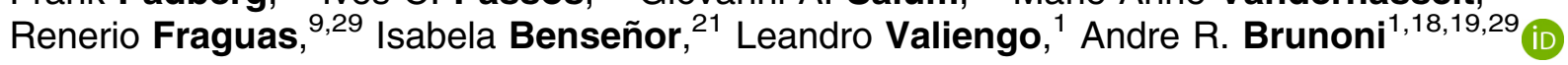

${ }^{1}$ Serviço Interdisciplinar de Neuromodulação, Laboratório de Neurociências (LIM-27), Departamento e Instituto de Psiquiatria, Hospital das Clínicas, Faculdade de Medicina, Universidade de São Paulo (USP), São Paulo, SP, Brazil. ${ }^{2}$ Centro de Neuropsicologia e Intervenção Cognitivo-Comportamental, Faculdade de Psicologia e Ciências da Educação, Universidade de Coimbra, Coimbra, Portugal. ${ }^{3}$ Department of Head and Skin, Faculty of Medicine and Health Sciences, Ghent University, Ghent, Belgium. ${ }^{4}$ Department of Psychiatry, University Hospital (UZ Brussel), Brussels, Belgium. ${ }^{5}$ Ghent Experimental Psychiatry (GHEP) Lab, Ghent University, Ghent, Belgium. ${ }^{6}$ Department of Electrical Engineering, Eindhoven University of Technology, Eindhoven, The Netherlands. ${ }^{7}$ Max Planck Institute of Psychiatry, Munich, Germany.

${ }^{8}$ Division of Interventional Cognitive Neurology, Department of Neurology, Berenson-Allen Center for Noninvasive Brain Stimulation, Beth Israel Deaconess Medical Center, Harvard Medical School, Boston, MA, USA. ${ }^{9}$ Laboratório de Neuroimagem em Psiquiatria (LIM-21), Departamento e Instituto de Psiquiatria, Hospital das Clínicas, Faculdade de Medicina, USP, São Paulo, SP, Brazil. ${ }^{10}$ Department of Psychiatry, University of Toronto, Toronto, ON, Canada. ${ }^{11}$ Centre for Addiction and Mental Health (CAMH), Toronto, ON, Canada. ${ }^{2}$ Spring Health, New York, NY, USA.

${ }^{13}$ Department of Psychiatry, Yale University, New Haven, CT, USA. ${ }^{14}$ Noninvasive Neuromodulation Unit, Experimental Therapeutic \& Pathophysiology Branch, National Institute of Mental Health, National Institutes of Health, Bethesda, MD, USA. ${ }^{15}$ Department of Psychiatry and Behavioral Sciences, School of Medicine, Duke University, Durham, NC, USA. ${ }^{16}$ Department of Psychiatry and Institute of Medical Science, Faculty of Medicine, University of Toronto, Toronto, ON, Canada. ${ }^{17}$ Centre for Mental Health and Krembil Research Institute, University Health Network, Toronto, ON, Canada. ${ }^{18}$ Laboratório de Neurociências (LIM-27), Departamento e Instituto de Psiquiatria, Hospital das Clínicas, Faculdade de Medicina, USP, São Paulo, SP, Brazil. ${ }^{19}$ Instituto Nacional de Biomarcadores em Neuropsiquiatria (INBioN), Departamento e Instituto de Psiquiatria, Hospital das Clínicas, Faculdade de Medicina, USP, São Paulo, SP, Brazil. ${ }^{20}$ School of Psychiatry and Black Dog Institute, University of New South Wales, Sydney, Australia. ${ }^{21}$ Estudo Longitudinal de Saúde do Adulto (ELSA), Centro de Pesquisa Clínica e Epidemiológica, Hospital Universitário, USP, São Paulo, SP, Brazil. ${ }^{22}$ Laboratório de Ressonância Magnética em Neurorradiologia (LIM-44) and Instituto de Radiologia, Hospital das Clínicas, Faculdade de Medicina, USP, São Paulo, SP, Brazil. ${ }^{23}$ Neurocognitive Research Laboratory, Department of Psychiatry, UT Southwestern Medical Center, Dallas, TX, USA. ${ }^{24}$ Wellcome Centre for Integrative Neuroimaging, Oxford Centre for Human Brain Activity, Department of Psychiatry, Warneford Hospital, University of Oxford, Oxford, United Kingdom. ${ }^{25}$ Department of Psychiatry and Psychotherapy, University Hospital, LMU Munich, Munich, Germany. ${ }^{26}$ Laboratório de Psiquiatria Molecular e Programa de Transtorno Bipolar, Hospital de Clínicas de Porto Alegre (HCPA), Programa de Pós-Graduação em Psiquiatria e Ciências do Comportamento, Universidade Federal do Rio Grande do Sul (UFRGS), Porto Alegre, RS, Brazil. ${ }^{27}$ Departamento de Psiquiatria, Seção de Afeto Negativo e Processos Sociais (SANPS), HCPA, UFRGS, Porto Alegre, RS, Brazil. ${ }^{28}$ Department of Experimental Clinical and Health Psychology,

Psychopathology and Affective Neuroscience Lab, Ghent University, Ghent, Belgium. ${ }^{29}$ Hospital Universitário, USP, São Paulo, SP, Brazil.

\begin{abstract}
Current first-line treatments for major depressive disorder (MDD) include pharmacotherapy and cognitive-behavioral therapy. However, one-third of depressed patients do not achieve remission after multiple medication trials, and psychotherapy can be costly and time-consuming. Although nonimplantable neuromodulation (NIN) techniques such as transcranial magnetic stimulation, transcranial direct current stimulation, electroconvulsive therapy, and magnetic seizure therapy are gaining momentum for treating MDD, the efficacy of non-convulsive techniques is still modest, whereas use of convulsive modalities is limited by their cognitive side effects. In this context, we propose that NIN techniques could benefit from a precision-oriented approach. In this review, we discuss the challenges and opportunities in implementing such a framework, focusing on enhancing NIN effects via a combination of individualized cognitive interventions, using closed-loop approaches, identifying multimodal biomarkers, using computer electric field modeling to guide targeting and quantify dosage, and using machine learning algorithms to integrate data collected at multiple biological levels and identify clinical responders. Though promising, this framework is currently limited, as previous studies have employed small samples and did not sufficiently explore pathophysiological mechanisms
\end{abstract}

Correspondence: André Russowsky Brunoni, Serviço Interdisciplinar de Neuromodulação, Rua Dr. Ovídio Pires de Campos, $785,2^{\circ}$ andar, Ala Sul, Instituto de Psiquiatria, CEP 05403-000, São Paulo, SP, Brazil.

E-mail: brunoni@usp.br

Submitted Oct 03 2019, accepted Dec 10 2019, Epub Mar 162020.
How to cite this article: Borrione L, Bellini H, Razza LB, Avila AG, Baeken C, Brem A-K, et al. Precision non-implantable neuromodulation therapies: a perspective for the depressed brain. Braz $\mathrm{J}$ Psychiatry. 2020;42:403-419. http://dx.doi.org/10.1590/1516-44462019-0741 
associated with NIN response and side effects. Moreover, cost-effectiveness analyses have not been performed. Nevertheless, further advancements in clinical trials of NIN could shift the field toward a more "precision-oriented" practice.

Keywords: Major depressive disorder; transcranial magnetic stimulation; transcranial direct current stimulation; electroconvulsive therapy; precision medicine

\section{Introduction}

Current psychiatric guidelines recommend several antidepressants and cognitive-behavioral psychotherapy (CBT) as first-line treatments for major depressive disorder (MDD). ${ }^{1,2}$ However, more than one-third of depressed patients will not achieve remission even after four adequate medical prescriptions of antidepressant drugs. ${ }^{3}$ Moreover, despite advances in psychopharmacology, even new medications can still produce several adverse effects that reduce tolerability and increase risk. ${ }^{4}$ Psychotherapy, in turn, is costly, time-consuming, does not suit all patients, and is not readily available in remote areas of the world. ${ }^{5}$

One possible explanation for the limited efficacy of mainstream antidepressant treatments is that they are typically applied in a "one-size-fits-all" and trial-and-error paradigm, with little biological guidance - i.e., information is mostly observational, with almost complete disregard for the specific neurobiological mechanisms underpinning the corresponding depressive symptomatology. To address this significant limitation in personalizing antidepressant treatments, a new field of "precision psychiatry" has been proposed, which aims to tailor medical treatment to the characteristics of each patient. ${ }^{6}$

Although this concept is not necessarily new (e.g., blood transfusion is "guided" by blood type examination), three new emerging tools ${ }^{6-8}$ are involved in the precision psychiatry framework: 1) incorporating the biological pathways of disease - in psychiatry, this is represented by the National Institute of Mental Health (NIMH) Research Domain Criteria (RDoc), a framework that evaluates mental illness at multiple clinical, endophenotypic, and neurobiological levels ${ }^{9}$; 2) multimodal big data collection, i.e., acquisition of clinical and biological data at scale, as exemplified by the opportunities presented by international consortiums such as the Enhancing Neurolmaging Genetics through Meta-Analysis (ENIGMA) ${ }^{10}$ and megacohorts such as the UK Biobank ${ }^{11}$; and 3) artificial intelligence for analysis of multidimensional and complex patterns in manifold data collected at multiple biological levels. ${ }^{12,13}$ Although precision psychiatry is still in its infancy, the continuous, rapid development of these tools will reshape clinical and research practice, enhancing treatment and minimizing adverse effects. ${ }^{6}$

Non-implantable neuromodulation (NIN) interventions, such as transcranial magnetic stimulation (TMS), transcranial direct current stimulation (tDCS), electroconvulsive therapy (ECT), and magnetic seizure therapy (MST), are non-pharmacological, non-psychotherapeutic interventions with distinct efficacy, safety, tolerability, and availability profiles. ${ }^{14-16}$ These techniques have been developed over multiple decades to bridge the efficacy and safety gaps of traditional antidepressant treatments, with concrete results. ${ }^{17}$

Nevertheless, major caveats remain, such as limited efficacy and significant adverse effects. In this context, the development of a "precision NIN" approach could both enhance clinical usability of NIN techniques (by improving efficacy and/or maximizing tolerability) and unveil their neurobiological mechanisms of action, which to date remain poorly understood. Additional challenges for precision NIN are the ability to combine them with other interventions ${ }^{18}$ and their spatiotemporal resolution, as the effects of NIN can be enhanced or decreased according to the site of application of the coils or electrodes and their synchronization with ongoing neuronal activity. ${ }^{19}$ Knowledge acquired from computer modeling and functional neuroimaging can be directed toward this purpose (Figure 1).

In this review, we present the concept of precision NIN as applied to antidepressant treatment. This framework would also be useful for other neuropsychiatric disorders. We first provide an overview of the state of the art and of the main NIN antidepressant modalities, and then present challenges and recent developments and opportunities of using NIN in the framework of precision psychiatry.

\section{Methods}

We convened a group of national and global leaders on the topics addressed in this review, such as MDD, neuroimaging, noninvasive brain stimulation, machine learning, neuropsychology, and precision psychiatry. These authors were invited to address specific parts of the manuscript, as well as to review its content as a whole. The PubMed, Google Scholar, and Web of Knowledge databases were searched from inception up to August 2019. Preference was given to recent comprehensive reviews, meta-analyses, pivotal randomized clinical trials that concerned NIN in the treatment of MDD, and highly cited articles in the field, with a view to offering an up-todate and comprehensive perspective from experts. We focused our review on clinical articles that investigated MDD.

\section{Non-implantable neuromodulation}

\section{Introduction and mechanisms of action}

NIN techniques use electrical or magnetic energy targeted at the brain ${ }^{20}$ (Figure 2). They do not require surgery, are less invasive, and involve less risk than implantable techniques, such as deep brain stimulation and vagus nerve stimulation. $^{22}$ They can be categorized into subconvulsive and convulsive modalities, the former also often described 


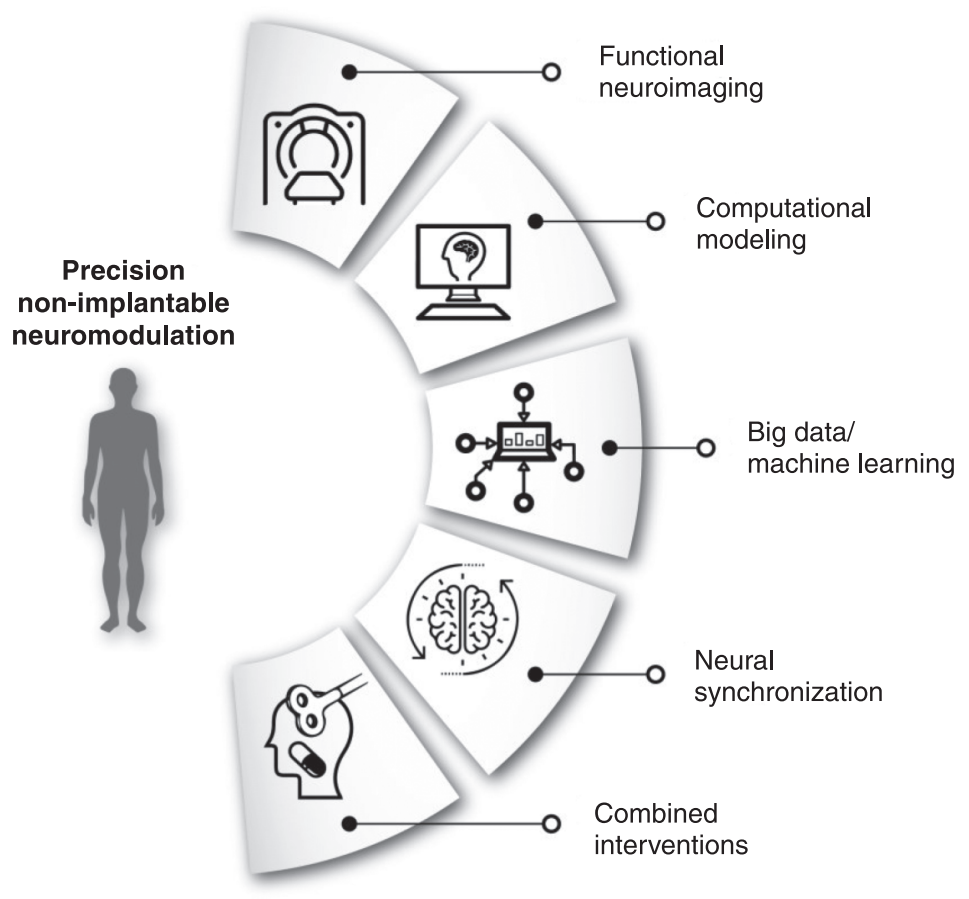

Figure 1 Precision non-implantable neuromodulation (NIN). In a precision NIN framework, advancements in related areas of research and knowledge directly influence treatment protocols (parameters such as stimulation site, timing, and dose, as well as combined behavioral/pharmacological interventions), aiming to increase individual antidepressant response.

as "non-invasive brain stimulation" (NIBS), which include TMS and tDCS, ${ }^{15}$ while the convulsive modalities include ECT and MST. ${ }^{23}$ Compared to the convulsive modalities, NIBS does not require sedation or anesthesia, and presents excellent safety and tolerability profiles. ${ }^{24-28}$

The neurobiological rationale supporting NIBS in depression is based on specific alterations of neurocircuitry function, which can be normalized by targeted stimulation of areas such as the dorsolateral prefrontal cortex (DLPFC) - the cortical area most commonly targeted, from early pilot studies to more recent, pivotal NIBS trials. ${ }^{29-33}$ Besides the practical convenience of safely targeting this area, the DLPFC is a key hub of the frontoparietal network (FPN), which has been implicated in the regulation of several processes, including decisionmaking, working memory, and attention, and is impaired in depression, ${ }^{34}$ particularly the left DLPFC. ${ }^{35}$ Hypoactivity of the FPN is associated with hyperactivity of the default mode network (DMN), which may promote depressive behaviors and cognitions such as negative bias, self-referential processing, and depressive rumination. ${ }^{36}$ The DLPFC is also a key node of the salience network (SN), which plays a key role in cognitive control (i.e., the self-regulation of thought, emotion, and behavior). ${ }^{37}$ Deficiencies in cognitive control and $\mathrm{SN}$ function and structure appear not only in depression, but transdiagnostically across a variety of Axis I disorders, ${ }^{38}$ suggesting that the mechanism of action of DLPFC-NIBS may be pertinent not only to depression, but to other forms of mental illness in which cognitive control is impaired.
NIBS to the DLPFC is thought to modulate the activity of this brain area, thus promoting an increase in FPN activity and a concomitant downregulation of DMN activity, leading to the improvement of depressive symptoms. ${ }^{39}$ Notably, anodal tDCS and high-frequency rTMS usually increase cortical excitability, although the net effect is also influenced by the underlying cortical activity. ${ }^{15}$ This rationale has been supported to some extent by neuroimaging studies in depressed patients receiving rTMS $^{21,40}$ and by recent validation studies. ${ }^{41}$

In turn, ECT and MST - both of which are performed in a controlled environment, under general anesthesia induce seizures via depolarization of neuronal networks. ${ }^{42}$ The mechanisms underlying the striking antidepressant effects associated with convulsive therapies remain poorly elucidated, ${ }^{43}$ and may include increased brain-derived neurotrophic factor (BDNF) levels, ${ }^{44}$ hippocampus and amygdala volumes, ${ }^{45,46}$ and hippocampal functional connectivity. ${ }^{47}$ Nonetheless, it is unclear whether the increase in hippocampal volume, a welldocumented effect, ${ }^{48}$ is an epiphenomenon or a necessary mechanism underpinning therapeutic ECT effects, as depression improvement is unrelated ${ }^{49}$ or even negatively associated ${ }^{48}$ with this outcome. The inflammatory theory associated with the convulsive NIN modalities is promising, as inflammatory cytokines decrease after ECT in depressed patients. ${ }^{50,51}$ As inflammation triggers the kynurenine pathway, leading to oxidative stress and serotonin depletion,52 rapid reduction of inflammation could mitigate depressive symptoms. ${ }^{53}$ 

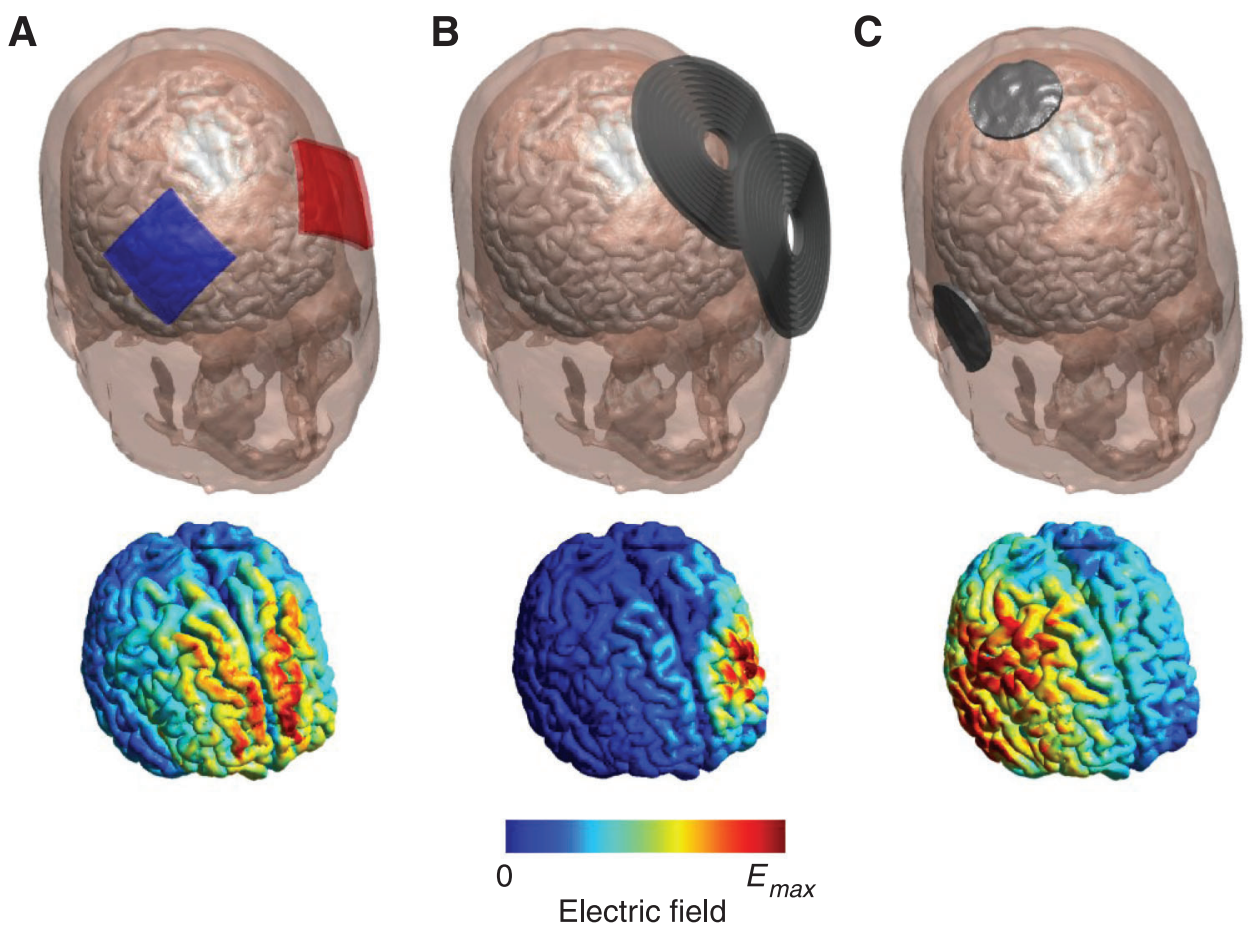

Figure 2 Examples of NIN techniques (top panel) and the corresponding electric field distribution in the brain (bottom panel): A) tDCS using $5 \times 5 \mathrm{~cm}$ electrodes placed over the bilateral DLPFC; electrodes are colored red and blue to distinguish anode (red) vs cathode (blue). B) TMS using the MagVenture B70 coil over the left DLPFC. C) Right unilateral ECT; conventional ECT uses a bipolar waveform and therefore does not distinguish between anodal vs. cathodal electrodes. Electric field strengths are normalized to their respective maximum value (Emax); absolute field strengths are very different across the modalities $(<1 \mathrm{~V} / \mathrm{m}$ for tDCS to $>100 \mathrm{~V} / \mathrm{m}$ for TMS and ECT). Figure produced using SimNIBS software. ${ }^{21}$ DLPFC = dorsolateral prefrontal cortex; $\mathrm{ECT}=$ electroconvulsive therapy; NIN = non-implantable neuromodulation; tDCS = transcranial direct current stimulation; TMS = transcranial magnetic stimulation.

\section{Transcranial magnetic stimulation}

TMS is based on the principle of electromagnetic induction via an electric alternating current passing through a coil. The magnetic field, which varies over time (1.0-2.5 Tesla), induces a secondary electric field, eliciting action potentials when targeting the underlying cortex. ${ }^{54}$ The original magnetic field passes through several layers (scalp, bone, meninges, etc.), with no resistance and deflection, inducing a relatively focal field. ${ }^{54}$

TMS can be delivered in various modalities - namely, high-frequency (HF, $5-20 \mathrm{~Hz}$ ), low-frequency (LF, $\leqslant 1 \mathrm{~Hz}$ ), theta-burst (TBS), and deep TMS (dTMS). ${ }^{21,55}$ Since the seminal study by Pascual-Leone et al. ${ }^{32}$ which showed efficacy of HF-rTMS over the left DLPFC, many trials have been performed. Of note, O'Reardon et al. randomized 301 patients with MDD without concomitant antidepressant use to receive either sham or active HFrTMS over the left DLPFC, and showed superior improvement in depression in the active group. ${ }^{30,56}$ This study provided pivotal data for FDA clearance of rTMS as antidepressant therapy. The effectiveness of HF-rTMS was further confirmed in two subsequent meta-analyses, with positive results both in accelerating clinical response to antidepressants ${ }^{57}$ and as monotherapy in unipolar and bipolar depression. ${ }^{58}$ Low-frequency rTMS (LF-rTMS) over the right DLPFC has demonstrated effectiveness in the treatment of MDD, ${ }^{59}$ with both $\mathrm{HF}$ and LF being regarded as first-line protocols. ${ }^{21}$ LF-rTMS can be especially advantageous when there is a high risk of seizures, poor tolerability to pain, or when the patient does not respond to HF-rTMS. ${ }^{55}$ Intermittent TBS, on the other hand, provides a new avenue for busy clinical services through exploration of more time-efficient rTMS protocols. A recent multicenter non-inferiority randomized clinical trial showed that intermittent theta-burst stimulation (iTBS), which lasts only 3 minutes, was non-inferior to 37.5 minutes of treatment with HF-rTMS. Both protocols were applied over the left DLPFC. ${ }^{29}$ Finally, deep rTMS with an $\mathrm{H}$ shaped coil has also demonstrated clinical effectiveness in MDD; this type of coil allows for deeper penetration of the magnetic field into the brain. ${ }^{55}$

Network meta-analyses have further demonstrated active rTMS to be superior to sham, albeit inferior to ECT. ${ }^{17,60}$ In these studies, it was found that priming, HFrTMS, LF-rTMS, bilateral rTMS, and TBS were more effective than placebo, although no active intervention was superior to any other.

Based on these results, rTMS is considered a first-line treatment for patients who have failed at least one trial with an antidepressant medication. ${ }^{21,61}$ On the other hand, the level of treatment resistance is known to be an important negative predictor of response to rTMS $^{62}$ Less is known regarding rTMS efficacy as a maintenance 
treatment in depression. In patients who have responded favorably to an acute course of rTMS, naturalistic studies have found that relapse is more common without any maintenance antidepressant strategy, ${ }^{63,64}$ while a metaanalysis evaluating durability of HF-rTMS showed that the antidepressant effects are small following a shorter acute treatment. ${ }^{65}$ Current recommendations have not reached a clear consensus regarding the most effective antidepressant maintenance protocol. ${ }^{21,61}$

Side effects of rTMS include the possible occurrence of mild headache and pain at the site of stimulation, although these symptoms typically resolve spontaneously and the treatment is well tolerated overall. ${ }^{55}$ The most serious side effect of rTMS is triggering of epileptic seizures, although this phenomenon is rare in clinical practice. In fact, seizures have been found to be extremely rare, and mainly occurred when rTMS protocols exceeded safety guidelines. ${ }^{24}$ Animal studies suggest that even at high intensities and prolonged exposure durations, there is little likelihood of damage to brain structures. ${ }^{66,67}$ Finally, the only absolute contraindication to rTMS is the presence of metallic and electronic material, such as cochlear implants, in close contact with the coil. ${ }^{55}$

\section{Transcranial direct current stimulation}

For tDCS, an electric current of low intensity (usually 1.0$2.5 \mathrm{~mA}$ ) is applied to the brain, via two electrodes placed over the scalp (anode and cathode), which is the most common protocol. ${ }^{68}$ The current passes through the skin, subcutaneous tissue, skull, and cerebrospinal fluid (CSF), and into the gray and white matter. Due to the impedance of the skull, only a fraction of the injected current reaches the brain. ${ }^{69}$ In addition, as the conventional spongeelectrode set is large (25 to $35 \mathrm{~cm}^{2}$ ) and the electrodes are placed relatively far apart on the head, the induced field is non-focal as the current flows from the anode to the cathode. ${ }^{28}$ The injected electrical current does not generate action potentials per se, but rather facilitates or inhibits synaptic transmission, respectively, via an increase or decrease in the frequency of action potentials in endogenous neuronal firing. ${ }^{69}$ For depression, tDCS montages employ anodal stimulation over the left DLPFC (with contralateral, variable cathode sites), thus aiming to counterbalance the hypoactivity of this brain area and subsequent hyperactivity of the $\mathrm{DMN} .^{70}$

tDCS is considered a safe and well tolerated technique, especially since the standard range of current intensities used does not induce brain injury. ${ }^{25,26,28}$ The most common side effects include itching and tingling at the scalp application sites. ${ }^{15}$ Skin burns are uncommon, and the risk can be further reduced with proper soaking of the electrodes, customized sponges, and adequate use of saline solution. ${ }^{71}$

Due to its portability and ease of use, tDCS has been investigated as an augmentative and substitute treatment for antidepressant medications. In a factorial study design, Brunoni et al. ${ }^{72}$ randomized 120 antidepressantfree depressed patients to receive placebo, sertraline only, tDCS only, or combined treatment with the two. ${ }^{72}$ The main study finding was that the combined treatment led to a faster and greater response compared to the other treatments. Subsequently, the same group ${ }^{31}$ designed a non-inferiority, sham-controlled design to compare tDCS vs. full-dose escitalopram. The study failed to show noninferiority of tDCS vs. escitalopram, although superiority analyses revealed that tDCS was more effective than placebo. Accordingly, recent meta-analyses have shown that tDCS is superior to placebo for response, remission, and depression improvement outcomes. ${ }^{73,74}$

Only three studies have investigated continuation of tDCS sessions after the acute treatment phase. ${ }^{75-77}$ All showed 6-month relapse rates varying from $25-50 \%$. Interestingly, the study that reported the lowest relapse rate had tDCS performed twice a week, ${ }^{76}$ whereas the one reporting higher relapse rates performed tDCS every other week. ${ }^{75}$ Taken together, this suggests an intensive tDCS treatment regimen is associated with lower relapse rates, although these studies were limited by small sample sizes and short follow-up periods.

While future randomized clinical trials involving tDCS in the treatment of depression should continue to investigate maintenance phase protocols, it would also be interesting to individualize the delivered dose using computer models, while evaluating the feasibility and safety of home-based sessions. ${ }^{70,78}$ Furthermore, electrical stimulation with different wave formats, such as transcranial alternating current stimulation (tACS) ${ }^{79}$ or transcranial random noise stimulation (tRNS), ${ }^{80}$ could be used to target MDD-related oscillatory brain activity in the DLPFC, possibly in combination with individualized neurofeedback strategies. ${ }^{81}$

\section{Convulsive modalities}

ECT delivers a stimulus of alternating polarity pulses, with an amplitude of 800-900 mA, via two electrodes placed on the scalp. ${ }^{82}$ Although the procedure is considered a second-line treatment for MDD due to the risk of cognitive side effects, it is regarded as a first-line treatment in some cases (e.g., MDD with acute suicidal or psychotic features). ${ }^{21,83}$ ECT is more effective than sham, antidepressant medications and psychotherapy, and rTMS, ${ }^{84}$ achieving very high response and remission rates. ${ }^{21}$

Historically, bitemporal (or bifrontotemporal) electrode positioning has been used, although right unilateral (RUL) placement has gained currency as a modality with relatively fewer cognitive side effects. ${ }^{82}$ In fact, there is a complex relationship between ECT "dose" (total charge delivered - a composite measure of current pulse amplitude, pulse width, frequency, and number of pulses indexed by the seizure threshold, ST) and electrode placement as a determinant of cognitive and antidepressant outcomes, which can be partly explained by electric field distribution. ${ }^{82}$ In the past, there was controversy as to the comparative efficacy of RUL vs. bitemporal ECT, the latter being considered more effective. However, recent meta-analyses have found that high-dose RUL and bitemporal ECT are equally effective, ${ }^{17,85}$ and considered that previous RUL ECT trials that used lower doses (e.g., less than six times the seizure threshold) might have underestimated its treatment effects. More recently, bifrontal ECT has been introduced as a form of ECT with 
efficacy comparable to that of bitemporal ECT, but fewer side effects. ${ }^{86}$ Maintenance-phase ECT (weekly, biweekly, or monthly sessions) is also considered effective and tolerable, with increased efficacy in association with medications, especially the combinations of nortriptyline and lithium ${ }^{87}$ and venlafaxine and lithium. ${ }^{88}$

Despite its effectiveness, ECT is limited due to the need for infrastructure (general anesthesia, trained personnel, and clinical evaluation), ${ }^{83}$ social stigma, ${ }^{89}$ and potential cognitive side effects, including postictal disorientation, anterograde amnesia, retrograde amnesia, and impairments in multiple other cognitive domains, including verbal fluency and executive function. ${ }^{21}$

The persistence, severity, and characterization of cognitive impairment remain topics of great debate in the current ECT literature, even after decades of research. ${ }^{90}$ Nevertheless, most acute ECT-related adverse events whether cognitive or non-cognitive - are mild, transient, and self-limited; more severe cardiovascular and neurological complications are rare, and can be managed through prophylactic and therapeutic measures. ${ }^{91}$

MST is a TMS variant that passes through the skull unimpeded and results in a more focused superficial electric field, concentrated in the cerebral cortex; hence, there is minimal stimulation of inner brain structures, such as the hippocampus. ${ }^{92}$ It delivers $25-100 \mathrm{~Hz}$ pulses for up to 10 seconds to trigger generalized seizures. ${ }^{23}$ Potentially, the more focal and limited electric field induced by MST could be associated with a lower incidence of cognitive side effects compared to ECT. ${ }^{93}$ Nevertheless, MST seizures show less robust ictal expression, postictal suppression, and generalization to the hippocampus compared to ECT. ${ }^{94}$ To date, studies that have compared MST and ECT found promising results, with MST having antidepressant effects comparable to those of RUL ECT and no cognitive side effects. ${ }^{23,95,96}$

\section{Challenges and opportunities for precision non-implantable neuromodulation}

NIBS methods are not yet mainstream treatments for depression. On the one hand, these techniques excel in safety and tolerability; on the other, they have modest antidepressant effects, are associated with variable costs, and not widely available. ${ }^{15,17,97} \mathrm{ECT}$, although highly effective, is limited by cognitive side effects and social stigma, while MST is currently experimental. To promote NIN applicability, we discuss the challenges and opportunities for increasing NIBS clinical effectiveness and decreasing ECT- and MST-related side effects, in a precision-oriented framework and in light of different forms of "target engagement" (a target being either a mechanism related to the disease or to the mode of action of the intervention itself). ${ }^{98}$

The "how": combining non-invasive brain stimulation with cognitive interventions

Evidence has demonstrated progressively that the neurobiological, behavioral, and antidepressant effects of
NIBS are dependent on the "state" of the targeted neural area at the time of stimulation. ${ }^{18}$

For example, in depression, patients exhibiting higher rostral anterior cingulate cortex (ACC) activity prior to stimulation showed a better antidepressant response to subsequent rTMS. ${ }^{99}$ This raises the prospect of experimentally controlled "pre-shaping" of brain states, induced by cognitive tasks and/or NIBS techniques, to more effectively target stimulation to redress neurobiological imbalances in depression. For instance, hyperconnectivity between the ACC and the medial prefrontal cortex (mPFC) has been linked to maladaptive depressive ruminations, and both CBT and rTMS, each alone, have been shown to downregulate this dysfunctional brain activity. ${ }^{18}$ Could pairing these interventions yield synergistic effects? In an interesting pilot study, concurrent rTMS and self-system therapy (SST, a modality similar to CBT) were performed in (albeit only five) depressed subjects, with positive results. ${ }^{100}$ Functional MRI was used to assess brain change in the left DLPFC (which was previously shown to be activated by SST-like tasks), and rTMS was then targeted at this individual area while an actual SST session was delivered. ${ }^{100}$ In a naturalistic, open-label, multicenter study, 196 depressed patients (most of whom were treatment-resistant) were assigned to receive CBT sessions with simultaneous $\mathrm{HF}(10 \mathrm{~Hz})$ or LF $(1 \mathrm{~Hz})$ rTMS. Response rates reached $66 \%$, with no difference between rTMS modalities. ${ }^{101}$

Regarding tDCS, a study in a rodent slice model showed that excitatory direct-current stimulation can strengthen cellular mechanisms thought to underlie learning and memory formation (long-term potentiation, LTP ${ }^{102}$ ). Critically, this enhancement occurred only when stimulation was applied "online," i.e., during LTP - there was no effect when the identical stimulation was applied "offline," i.e., prior to LTP. Behaviorally, parallel findings in rodents and humans showed that excitatory tDCS during learning enhanced memory for what was learned. ${ }^{103}$ Such enhancement has been shown to depend critically on stimulation during learning - the same stimulation applied prior to learning can have null or even antagonistic effects. ${ }^{104}$ Such basic neuroscience work suggests that the clinical efficacy of tDCS could potentially be enhanced if it is applied during learning that is designed to promote positive mood change, e.g., CBT (so-called functional targeting). ${ }^{105}$

The combination of tDCS with psychotherapy is particularly appealing. As both interventions target the prefrontal cortex, their combination might result in a positive synergy, with tDCS potentially enhancing a range of cognitive processes recruited during psychotherapy. ${ }^{106}$ Various forms of psychotherapy have been combined with tDCS. In an early, open-label study, Martin et al. combined tDCS with a task designed to improve identification of emotional states, in treatment-resistant depressed participants, with positive results $(41 \%$ of study completers displayed treatment response). ${ }^{107}$ In another recent pilot study, ${ }^{108}$ patients with treatment-resistant depression (TRD) received active tDCS (20 min, $2 \mathrm{~mA}$, applied to the left DLPFC) on 8 consecutive days and were randomly assigned to receive either 2 hours of mindfulness-based cognitive therapy (MBCT) or a 30-minute relaxation 
session immediately after each tDCS session. Results indicate a longer lasting reduction of depressive symptoms and enhanced cognitive processes in patients receiving the tDCS/MBCT combination. An ongoing, multicenter study is evaluating the efficacy of tDCS combined with group CBT. ${ }^{109}$ In this study, 192 depressed patients are being randomized to 12 sessions of either: 1) CBT + active tDCS (2 mA, 30 minutes); 2) CBT + sham tDCS; or 3) CBT alone. ${ }^{109}$

The combination of tDCS with DLPFC activation techniques, such as working memory training or combined cognitive training, has also shown promising results in healthy subjects, ${ }^{110}$ schizophrenia, ${ }^{111}$ mild cognitive impairment, ${ }^{112}$ and cognitive impairment in Parkinson's disease. ${ }^{113}$ For combined treatment, tDCS has advantages over rTMS: it does not produce noise, which can be a distracting factor in rTMS sessions, and is portable. Cognitive remediation, such as the training of specific cognitive control processes, can also be performed following an intervention with TMS or ITBS in a sequential fashion, where cognitive remediation would be timed to take advantage of the enhanced cognitive capacities provided by the NIBS intervention. In this direction, an ongoing trial (PACt-MD) is comparing the efficacy of tDCS combined with cognitive remediation vs. double placebo in slowing cognitive decline and preventing Alzheimer's disease in older persons with mild cognitive impairment, or MDD with or without mild cognitive impairment (PACtMD, ClinicalTrials.gov, number NCT02386670).

Furthermore, given that other forms of psychotherapy, such as interpersonal therapy, have been found to be effective in the treatment of MDD, ${ }^{114}$ it would also be interesting to study the combination of these techniques with NIN.

The "when": combining NIBS with real-time neuroimaging and electrophysiology

The neurobiological effects of NIBS can also be assessed during or after application sessions, ${ }^{115-117}$ in what have been termed "online" and "offline" approaches, respectively. ${ }^{115}$

The "online" approach allows use of imaging techniques to quantify local neural network properties and application of NIBS so as to interfere with ongoing neuronal processing, visualizing how NIBS modulates the level or timing of neuronal activity with imaging and electrophysiology. ${ }^{115}$ For instance, a few studies have used fMRI to evaluate the online propagation of TMS-induced effects targeted to the prefrontal cortex. ${ }^{118-120}$ Further studies are required to explore this propagation as a potential biomarker for rTMS efficacy in the treatment of depressive patients.

In the "offline" approach, one can increase or decrease the excitability of a brain region and measure the consequences thereof (i.e., with tDCS). ${ }^{115}$ For instance, a recent study of volunteers with high trait anxiety showed, through fMRI observation, that a single session of the typical tDCS protocol used in depression suppressed hyperactive fear signaling in the amygdala and increased activity in frontoparietal attentional top-down control regions. ${ }^{121}$
By these approaches, different combinations of NIBS modalities can be synchronized with neural oscillatory network activities, through real-time EEG or fMRI readouts which are further analyzed, thus closing the loop between stimulation and neurobiological response. ${ }^{116}$

\section{The "where": positioning and dose quantification}

Optimal coil/electrode positioning is important to decrease within- and between-subject heterogeneity in the induced electric field and enhance clinical efficacy. Methods for standardizing coil/electrode positioning are commonly used, such as scalp landmark or hotspot-based coil placement for rTMS, ${ }^{122}$ or headgear that secures the electrodes in the desired location. ${ }^{123}$ Studies have shown that even small changes in coil/electrode positioning can change the induced neurobiological effects and negatively affect clinical outcomes. ${ }^{124,125}$ More sophisticated targeting approaches use individual or group-level anatomical and/or functional imaging to define the stimulation site, including the possible use of multiple electrodes to stimulate wider brain networks in a multifocal approach. ${ }^{126}$ Targeting based on fMRI guidance has been shown to produce stronger online rTMS effects compared to other targeting strategies. ${ }^{127}$ The use of neuronavigation systems can greatly improve the spatial precision of TMS. Furthermore, robotic coil-holder systems can provide millimeter accuracy and continuous tracking of the TMS coil. One such robotic system recently received FDA 510(k) clearance.

"Dose" quantification is key to determine the doseresponse gradient and to titrate the intervention parameters accordingly. Nonetheless, determining dose is challenging in NIN, as various stimulation parameters are employed (e.g., current intensity, waveform, and duration for electrical stimulation; number, frequency, pattern, and intensity for magnetic stimulation; frequency and duration of treatment course), which influence one another in complex interactions. ${ }^{\text {.28 }}$ The net result (excitation, inhibition, or no effect) is influenced by other concomitant interventions (pharmacological agents, cognitive tasks, other NIBS interventions, and psychological interventions), ${ }^{26,129}$ as well as by network activity (i.e., brain state). For convulsive therapies, there might be a trade-off between using higher doses to produce greater clinical benefits but with additional side effects. ${ }^{85}$

More recently, computational models have been used to quantify electric fields (EFs) in brain regions of interest (ROls). In fact, there are freely available software packages that perform electric field simulations using high-quality MRI images (templates or individualized) via a series of steps: 1) automated tissue segmentation of structural MRI; 2) meshing of the different tissue compartments to form a 3D model of the head and brain; 3) processing DTI data to extract white-matter anisotropic conductivity values; 4) incorporating electrodes and the TMS coil on the head model; 5) assigning appropriate electrical properties for the tissues and electrode/coil; 6) solving for the electric field and current density, via numerical methods such as finite element or boundary element methods; and 7) exporting, visualizing, and/or 
transforming electric field distribution to standard space for group analysis. ${ }^{130}$ The resulting EFs can be graphically assessed; the results of EFs per ROI can be printed out and mapped into standard spaces, such as MNI and FreeSurfer, for analysis of correlations between current distribution, clinical outcomes, and structural and functional neuroimaging findings. These computational models allow researchers to 1) understand the biophysics and mechanism of action for NIN modalities; 2) benchmark and compare different technologies; and 3) quantify interindividual variation in the induced dose as it relates to clinical outcome. Further work is needed to investigate the effects of EFs on brain tissue, and whether models can be used for treatment planning and optimization.

\section{The "who": identifying responders}

The predictors of NIN response are mostly unknown. Although treatment resistance in MDD is a robust clinical predictor of poor response for most antidepressant strategies, including NIN interventions, ${ }^{131-133}$ this might be related more to the depressive episode per se than to the intervention. In addition, the absence of improvement in response to rTMS early during treatment predicts continued non-improvement with further rTMS treatment, ${ }^{134}$ whereas acute improvement with ECT predicts final remission. ${ }^{135}$

Other predictors have shown mixed results. For instance, in tDCS, higher "dose" was associated with better outcomes in one meta-analysis, ${ }^{131}$ but a further sham-controlled trial using a higher dose than previous ones yielded nonsignificant findings. ${ }^{136}$ Another study that applied tDCS over the motor cortex also concluded that enhancement of tDCS "dose" does not necessarily increase the neurobiological effects of stimulation, but might shift the direction of excitability alterations. ${ }^{137}$ Furthermore, tDCS responders have been found to display greater improvements in the MADRS dysphoria and retardation factors compared to nonresponders. ${ }^{138}$ For rTMS, although most research to date has focused more on testing the efficacy of different interventions rather than on identifying subgroups of patients who would respond better to a particular intervention, ${ }^{139}$ some recent work suggests that the treatment may be most effective for certain particular "biotypes" of depression, detectable from whole-brain network connectivity on functional MRI, ${ }^{140}$ and that the optimal rTMS parameters to achieve antidepressant effect might vary depending on treatment resistance, age, and sex. ${ }^{141}$ Moreover, greater response to a LF-rTMS protocol has been associated with lower MADRS retardation scores at baseline. ${ }^{142}$ Regarding ECT, even first-line recommendations such as older age, psychosis, and melancholic features ${ }^{21}$ were not consistently identified in a meta-analysis. ${ }^{133}$ One study reported that ECT responders displayed higher scores on a MADRS dysphoria factor compared to nonresponders, while the procedure had only a small effect on a MADRS vegetative factor. ${ }^{143}$

Although relatively large trials have been conducted in the NIN field, ${ }^{29,31,144}$ to the best of our knowledge, there is no published research using machine-learning algorithms to predict NIN response based on a clinical dataset - as has already been done in pharmacological trials. ${ }^{145}$ For example, using a gradient boosting model, Chekroud et al. ${ }^{145}$ identified in STAR ${ }^{\star} \mathrm{D}$ a dataset of 25 variables that predicted depression response significantly above chance. In another STAR ${ }^{\star} D$ analysis, Chekroud et al. ${ }^{146}$ identified which pharmacotherapies would be associated with greater depression improvement for patients grouped according to a cluster of depressive symptoms. These approaches are important and fundamentally different from statistical approaches to identification of predictors, which are based on groups, not individuals. ${ }^{13,147}$ In fact, statistical methods focus on inference - creating a mathematical model that tests a hypothesis about how a system behaves, whereas machine learning focuses on prediction - i.e., finding generalizable predictive patterns that aim to forecast future behaviors regardless of their mechanistic basis ${ }^{12}$ (Figure 3). Additionally, through employing almost no pre-assumptions and a nonlinear function canvas, machine learning techniques can model complex patterns that can identify relationships between large amounts of data and data of diverse types, ${ }^{148,149}$ increasing the processing speed and output of predictive models. For instance, a machine-learning modality known as "deep learning" provides a promising approach for analysis of the relationship between electromagnetic fields and biological tissues (i.e., a head model is automatically generated through $\mathrm{MRI}$, with correspondence between voxels to specific tissue types with given electrical conductivity values). ${ }^{150}$

One concern about such approaches is the lack of interpretability that the resulting models usually possess. There is no clear way of interpreting complex nonlinear models. In many clinical applications, including selection of treatment or prediction of side effects, the clinician does not need to fully comprehend how the machine is processing information. In that case, the main concern is how effectively the model can predict a specific outcome.

Preliminary work from our group (under review) used data from the ELECT-TDCS ${ }^{31}$ to estimate single-subject prediction of treatment response to tDCS, escitalopram, or placebo. A total of 245 subjects were included, of whom $55 \%$ were women $(n=166)$ and $29 \%$ had TRD $(n=91) .{ }^{31}$ The feature dataset included baseline clinical, sociodemographic, somatic, treatment-related, and depression-related variables, as well as mood and anxiety scales. Using a XGBoost tree boosting algorithm, we could predict response to placebo, escitalopram and tDCS with $45 \%(95 \% \mathrm{Cl} 39-52), 56 \%(50-61 \%)$, and $67 \%$ (62-71\%) balanced accuracy, respectively. This preliminary work reveals that ML-based approaches can predict NIBS response above chance, facilitating further investigation of this approach in upcoming studies using clinical and biological data.

The identification of responders could potentially be greatly enhanced by using biomarkers. ${ }^{151}$ For precision NIN, biomarkers of treatment response would be especially useful, not only due to their predictive value but also particularly - to shed light on the mechanisms of action of NIN. Importantly, biomarkers of disease might not be necessarily related to treatment response, and a biomarker for a pharmacological treatment might not necessarily 
A

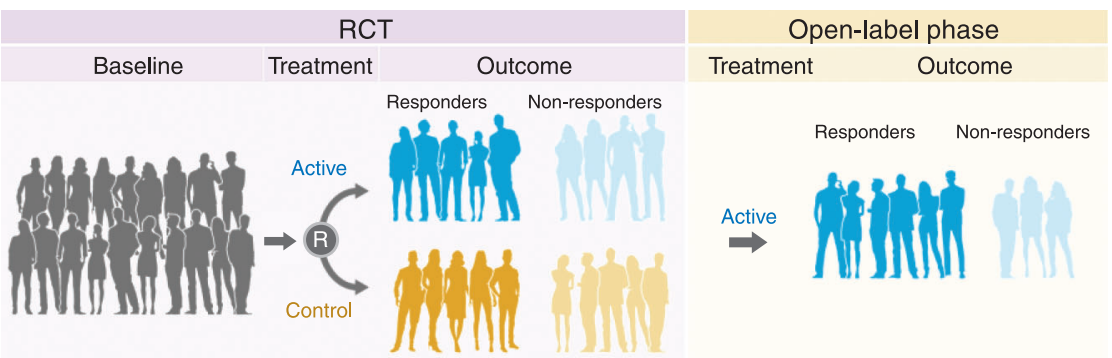

B

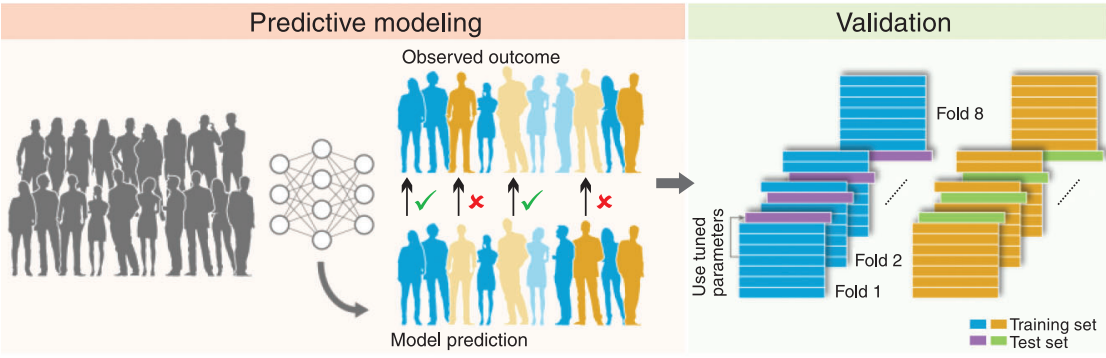

C

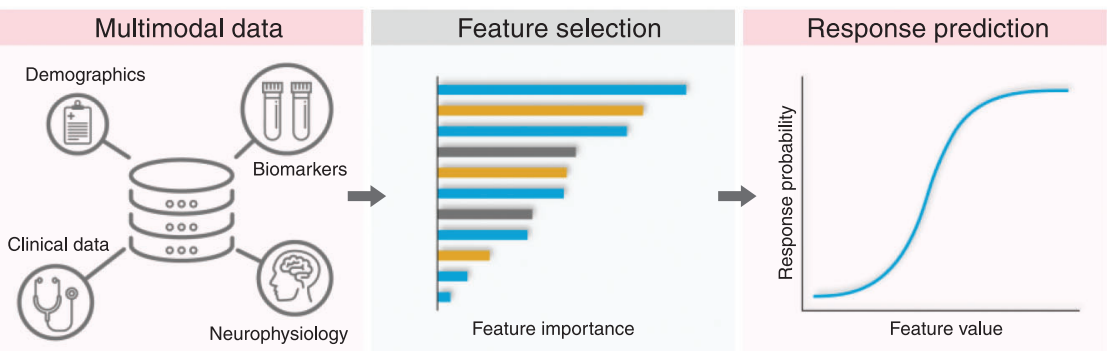

Figure 3 Example of a machine learning pipeline. Analysis pipeline. A) Treatment outcomes of group are predicted according to the feature dataset. B) Models are trained to classify responders and non-responders at the study endpoint. Performance is evaluated in a repeated nested cross-validation paradigm. C) Features with the highest contribution to the model can be identified. $\mathrm{RCT}=$ randomized clinical trial.

apply to NIN. Among several potential biomarkers, we propose that cognitive, neuroimaging, and neurophysiological markers would be particularly useful for precision NIN, since they are directly targeted by these techniques.

Structural and functional neuroimaging biomarkers have indicated that the volume and thickness of certain structures (e.g., portions of the prefrontal cortex and anterior cingulate cortex), the resting-state connectivity of certain networks, and the connectivity between ROls predict and are modified by the antidepressant effects of NIBS. ${ }^{152-155}$ For instance, a recent study showed that patients respond bimodally to rTMS: at the beginning of treatment, nonresponders exhibited higher anhedonia and lower connectivity in a brain network classically associated with reward, consisting of the ventral tegmental area, striatum, and part of the VMPFC. This study indicated that a subtype of depressive patients, identified on the basis of syndromic and neuroimaging characteristics, may respond better to TTMS $^{156}$ Also looking at treatment-response biomarkers, researchers found early response to rTMS treatment to be predictable by the integrity of an extended salience-executive system, indexed by fronto-insular connectivity and $\mathrm{SN}$ connectivity with visual processing regions, although this was not true for sustained response at 3-month follow-up. ${ }^{157}$ In an exploratory analysis, researchers found that higher functional connectivity between the DLPFC and striatum predicted better treatment response to TMS in a group of depressed patients. ${ }^{158}$ In another study ${ }^{140}$ that used functional magnetic resonance imaging (fMRI) in a large, multisite sample of 1,188 depressive patients, four distinct neurophysiological subtypes ("biotypes") were identified on the basis of distinct patterns of dysfunctional connectivity in frontostriatal and limbic networks. Patients in "biotype 1" were approximately three times more likely to benefit from rTMS over the DMPFC than those in "biotypes" 2 or $4,{ }^{140}$ although these findings need to be interpreted with caution, given questions about their replicability. ${ }^{159}$ For tDCS, a recent study showed that larger gray-matter volumes in the left DLPFC at baseline were further associated with antidepressant response to tDCS, but not to escitalopram or placebo. ${ }^{160}$ Although the effect sizes were small and had no individual-level predictive value, these findings contribute to our understanding of the antidepressant effects of tDCS by showing a specific association between the stimulated area and further antidepressant response. So far, there does not seem to be a consistent unique pattern of functional or structural abnormality to predict the effect of noninvasive neuromodulatory MDD interventions. In future, combined use of biomarkers may help guide treatment selection. ${ }^{161}$ 
Cognitive functions are another set of biomarkers worth exploring in NIN trials. In depression, these are often impaired independently of mood ${ }^{162-164}$ and are associated with network dysfunctions. ${ }^{165,166}$ With the exception of ECT, NIBS appears to be cognitively safe in healthy adults, ${ }^{25,167}$ with most single-session studies indicating no cognitive decline, although exceptions have been noted. ${ }^{168,169}$ In depressed patients, recent metaanalyses found that NIBS techniques are not associated with cognitive side effects. ${ }^{170,171}$ In contrast, cognitive adverse effects are common in convulsive therapies. ${ }^{91}$ NIBS interventions could be designed to not only improve mood, but to concomitantly induce cognitive enhancement. The implementation of such a combined approach would depend upon multiple factors, including NIBS protocols, specific neurocircuitry and physiological premises, online and offline stimulation, pre-existing cognitive difficulties, and other clinical and demographic factors. For instance, NIBS administered over the PFC induced improvement in a working memory task. ${ }^{172}$ Thus, it can be supposed that, in MDD, PFC stimulation could exert pro-cognitive effects, particularly in complex attention and working-memory domains. ${ }^{173}$ Nevertheless, although some studies suggested cognitive improvement in some tasks after rTMS in depressive patients, the majority of studies showed no cognitive benefits after NIBS. ${ }^{174}$ Possible reasons for null findings are limitations of specific NIBS paradigms (e.g., poor spatial targeting, inadequate dose), practice effects, reduced sensitivity and specificity of the tests (e.g., paper-and-pen instead of computerized tests), ceiling effects, ${ }^{174,175}$ and the lack of concomitant cognitive activity. Cognitive functions could also be leveraged to individualize treatment approaches and predict treatment outcome. For example, baseline cognitive performance or acute cognitive effects after the first NIN session can predict antidepressant response to NIN,${ }^{176,177}$ and could therefore be used as a potentially straightforward method for prediction in combination with machine-learning approaches. Cognitive functions have also been shown to be useful predictors for outcomes of other treatment approaches, such as psychotherapy, ${ }_{178}$ and more long-term outcomes, such as return to work. ${ }^{179}$ Moreover, evaluating cognitive changes can provide mechanistic insights into the antidepressant mechanisms of action of NIN - e.g., by exploring whether they moderate and/or mediate depression improvement - and into NIN-induced changes in specific brain structures. ${ }^{180}$ In this case, cognitive changes have been operationalized as fundamental mechanisms of action, but also as more translational processes, such as self-referential thoughts and emotions (e.g., negative affect, rumination, regret, cognitive bias). To date, most of this research is being performed in healthy volunteers, but the transition to clinical samples - also based on the idea of functional targeting of similar functional and neuroanatomical circuits using multimodal interventions - is slowly moving forward.

Motor cortical excitability (MCE) measures were the first neurophysiological biomarkers investigated in MDD, as the motor cortex can be easily probed using singleand paired-pulse TMS, which are associated with GABA and glutamate activity in this structure. ${ }^{181,182}$ Studies have shown that baseline measures of cortical inhibition and facilitation were associated with antidepressant response to tDCS, rTMS, and ECT, ${ }^{31,183,184}$ although effect sizes were small. In addition, EEG-based neurophysiological parameters are associated with antidepressant response to rTMS. ${ }^{185,186}$ More recently, it has been advocated that prefrontal excitability indexed using TMSevoked potentials and TMS-EEG systems might be more a specific marker of NIN effects compared to MCE, as these methods can probe the cortical excitability of frontal brain areas implicated in depressive pathophysiology, with high temporal resolution. ${ }^{187}$ However, these techniques are still novel and technically challenging, and it is still unclear which indexes better represent GABA activity. ${ }^{116}$ These limitations notwithstanding, promising findings have been observed using this biomarker modality to predict NIN response. ${ }^{188-190}$

Other biological markers have also been explored in NIN, including genetic and non-genetic peripheral biomarkers and heart rate variability. ${ }^{44,191-199}$ Although some positive findings were found, results have been inconsistent, and mostly derived from open studies. In addition, the identification of candidate genes has been challenged by more recent studies showing that most previous findings are likely to be false positives. ${ }^{200}$ Likewise, the literature on peripheral depression biomarkers is fraught with bias. ${ }^{201,202}$

\section{Limitations and perspectives}

Although promising, the precision NIN framework should be pursued and expanded with a view to improved clinical applicability. For instance, despite the investigation of several biomarkers, most positive findings have emerged from poorly controlled exploratory studies using small sample sizes, thus requiring further validation. In this regard, properly controlled studies of biomarkers could further expand our knowledge of their role in the pathophysiological processes related to MDD and help predict response to treatment. Strategies that would enhance biomarker validity include adequately powered sample sizes and a priori hypotheses for the role of the markers of interest. Novel clinical trials investigating NIN interventions should embed the investigation of biomarkers in their design. For certain NIN modalities in which clinical efficacy is already proven, such as most variants of rTMS and ECT, sham-controlled trials are not necessary and, in fact, not feasible from an ethical perspective, as equipoise to placebo cannot be assumed. Therefore, academic centers that perform rTMS and ECT should incorporate systematic data collection of clinical and demographic characteristics - as well as questionnaires, inventories and scales measuring depression and cognitive changes during treatment - into their clinical routine. Ideally, molecular and neuroimaging biomarkers should be collected as well, and data could be shared by different data centers. Such an approach is exemplified by the Global ECT-MRI Research Collaboration, which already includes more than 22 centers collecting ECT and MRI data worldwide. ${ }^{48}$ On the other hand, for other NIN 
interventions, such as tDCS and MST, phase-3 controlled studies are still necessary, as clinical efficacy remains unproven. For such trials, we recommend that investigators incorporate a comprehensive set of biomarkers to be investigated within the context of the primary study hypothesis. In this context, the framework of "target engagement" and "target validation" is useful - i.e., those biomarkers deemed most promising on the basis of preclinical and early clinical findings should be investigated as predictors and moderators of clinical response.

Likewise, even though the logic of using approaches such as machine-learning in individual patient data and meta-analytic datasets is sound and necessary, any findings would still be retrospective, requiring further validation in novel datasets distinct from those on which the classifiers were trained, to determine generalizability. This necessitates a global effort in scientific collaboration and data sharing, with particular focus on overcoming difficulties in access to the literature and primary data held beyond paywalls. In this sense, open-access initiatives are welcome, as are promising changes in business models of academic publishing. In this context, one of the leading journals of the field (Brain Stimulation) has become fully open-access as of January 1,2020.203 Moreover, policies ensuring that properly anonymized data from clinical trials (whether sponsored by public or private institutions) can be shared under request should be endorsed by regulatory agencies to further promote data-sharing initiatives.

Machine learning-guided intervention trials in NIN are still a necessary second step to further validate predictive algorithms. ${ }^{204}$ Cost-effectiveness analyses should also be performed to verify whether a precision-oriented approach is economically advantageous: on the one hand, enhancing efficacy and decreasing side effects can increase individual benefits and reduce treatment cost; on the other, the additional costs of using precision techniques should be considered. For instance, the advantages of tDCS include its low cost and portability, but the neuroimaging scans and individualized electrode positioning required before treatment could make these advantages moot if additional gains in efficacy and efficiency are not achieved. Although not discussed in the present review, computational and preclinical studies would be useful to deepen our understanding of NIN techniques, ${ }^{205}$ while studies in healthy volunteers are needed to narrow the parameter spaces of these techniques (for instance, by using closed-loop bayesian, adaptive optimization). ${ }^{206}$

Finally, it should be noted that the selection of studies for this special article was unsystematic, i.e., publications were deemed relevant and selected according to the perspective of the authors, with the inherent subjective limitations that such a narrative entails.

\section{Conclusions}

Depressive disorders are prevalent, disabling conditions. Conventional antidepressant treatments fail to induce remission in approximately one-third of patients with MDD, may result in intolerable side effects (first-line medications), or may be expensive and time-consuming (psychotherapy). Moreover, such therapies are still prescribed on a "trial-and-error" basis, in which achievement of a satisfactory response can take several months.

In this context, NIN techniques are increasingly considered safe, tolerable, and effective, whether as monotherapy or augmented with other interventions, such as medications and psychotherapy. Furthermore, as stimulation parameters can be directed to specifically affected brain areas, NIN is also undergoing a paradigm shift towards a precision-oriented framework that takes into consideration "knowledge about brain circuits that underlie complex cognitive, emotional and self-reflective functions" in order to guide individualized patient-oriented treatments. ${ }^{36}$ Ultimately, this new framework does not rely solely on observable clinical outcome information, but also on data from multiple biological levels, from cells to circuits. As open-access initiatives across the globe give space to merging and analyzing large datasets and subjects in clinical trials are increasingly assessed via multimodal approaches, a greater understanding of methods for handling big data will be mandatory for specialists in the field.

In this context, future NIN-related research would benefit from a focus on optimization of its parameters, discovery of remission- and response-related biomarkers, elucidation of cognitive safety and enhancement mechanisms, and advancement of scientific knowledge related to mechanisms of NIN action.

\section{Acknowledgements}

AGA is supported by Fundação para a Ciência e Tecnologia and Programa COMPETE, Portugal (grant PTDC/ MHC-PAP/5618/2014 [POCI-01-0145-FEDER-016836]; http://www.poci-compete2020.pt/). Z-DD is supported by the National Institute of Mental Health Intramural Research Program (grant ZIAMH002955) and by a Young Investigator Award from the Brain \& Behavior Research Foundation (grant 26161). SMM receives research support from the National Institutes of Health (NIH) and is a consultant to Pearson Assessment. JO'S is supported by a Sir Henry Dale Fellowship jointly funded by the Wellcome Trust and the Royal Society (grant $215451 / Z / 19 / Z$ ). ICP is supported by funding from Secretaria Nacional de Políticas sobre Drogas (SENAD) and Conselho Nacional de Desenvolvimento Científico e Tecnológico (CNPq). LBR is supported by Fundação de Amparo à Pesquisa do Estado de São Paulo (FAPESP; grant 2019/07256-7). ARB is supported by productivity grants from CNPq-1B and the Programa de Incentivo à Produtividade Acadêmica (PIPA), Faculdade de Medicina, Universidade de São Paulo (USP).

\section{Disclosure}

Z-DD is listed as inventor on patents/patent applications related to brain stimulation technology, assigned to Columbia University and NEVA Electromagnetics, not licensed, and with no remuneration. Although he is an employee of the U.S. government, the views expressed 
are his own and do not necessarily represent the views of the National Institutes of Health, the Department of Health and Human Services, or the U.S. government. The other authors report no conflicts of interest.

\section{References}

1 Kennedy SH, Lam RW, McIntyre RS, Tourjman SV, Bhat V, Blier P, et al. Canadian Network for Mood and Anxiety Treatments (CAN MAT) 2016 clinical guidelines for the management of adults with major depressive disorder: section 3. Pharmacological treatments. Can J Psychiatry. 2016;61:540-60.

2 Parikh SV, Quilty LC, Ravitz P, Rosenbluth M, Pavlova B, Grigoriadis S, et al. Canadian Network for Mood and Anxiety Treatments (CANMAT) 2016 clinical guidelines for the management of adults with major depressive disorder: section 2. Psychological treatments. Can J Psychiatry. 2016;61:524-39.

3 Rush AJ, Trivedi MH, Wisniewski SR, Nierenberg AA, Stewart JW, Warden $D$, et al. Acute and longer-term outcomes in depressed outpatients requiring one or several treatment steps: a STAR ${ }^{\star} D$ report. Am J Psychiatry. 2006;163:1905-17.

4 Carvalho AF, Sharma MS, Brunoni AR, Vieta E, Fava GA. The safety, tolerability and risks associated with the use of newer generation antidepressant drugs: a critical review of the literature. Psychother Psychosom. 2016;85:270-88.

5 Keller MB, McCullough JP, Klein DN, Arnow B, Dunner DL, Gelenberg AJ, et al. A comparison of nefazodone, the cognitive behavioral-analysis system of psychotherapy, and their combination for the treatment of chronic depression. N Engl J Med. 2000;342: 1462-70.

6 Fernandes BS, Williams LM, Steiner J, Leboyer M, Carvalho AF, Berk M. The new field of "precision psychiatry." BMC Med. 2017;15:80.

7 Jameson JL, Longo DL. Precision medicine -- personalized, problematic, and promising. N Engl J Med. 2015;372:2229-34.

8 Chekroud AM, Lane CE, Ross DA. Computational psychiatry: embracing uncertainty and focusing on individuals, not averages. Biol Psychiatry. 2017;82:e45-7.

9 Insel T, Cuthbert B, Garvey M, Heinssen R, Pine DS, Quinn K, et al. Research domain criteria (RDoC): toward a new classification framework for research on mental disorders. Am J Psychiatry. 2010;167:748-51.

10 Thompson PM, Stein JL, Medland SE, Hibar DP, Vasquez AA, Renteria ME, et al. The ENIGMA Consortium: large-scale collaborative analyses of neuroimaging and genetic data. Brain Imaging Behav. 2014;8:153-82.

11 Sudlow C, Gallacher J, Allen N, Beral V, Burton P, Danesh J, et al. UK biobank: an open access resource for identifying the causes of a wide range of complex diseases of middle and old age. PLoS Med. 2015;12:e1001779.

12 Bzdok D, Meyer-Lindenberg A. Machine learning for precision psychiatry: opportunities and challenges. Biol Psychiatry Cogn Neurosci Neuroimaging. 2018;3:223-30.

13 Dwyer DB, Falkai P, Koutsouleris N. Machine learning approaches for clinical psychology and psychiatry. Annu Rev Clin Psychol. 2018; $14: 91-118$

14 Brunoni AR, Teng CT, Correa C, Imamura M, Brasil-Neto JP, Boechat R, et al. Neuromodulation approaches for the treatment of major depression: challenges and recommendations from a working group meeting. Arq Neuropsiquiatr. 2010;68:433-51.

15 Brunoni AR, Sampaio-Junior B, Moffa AH, Aparicio LV, Gordon P, Klein I, et al. Noninvasive brain stimulation in psychiatric disorders: a primer. Braz J Psychiatry. 2019;41:70-81.

16 Baeken C, Brem AK, Arns M, Brunoni AR, Filipčić I, Ganho-Ávila A, et al. Repetitive transcranial magnetic stimulation treatment for depressive disorders: current knowledge and future directions. Curr Opin Psychiatry. 2019;32:409-15.

17 Mutz J, Vipulananthan V, Carter B, Hurlemann R, Fu CH, Young $\mathrm{AH}$. Comparative efficacy and acceptability of non-surgical brain stimulation for the acute treatment of major depressive episodes in adults: systematic review and network meta-analysis. BMJ. 2019;364:I1079.
18 Sathappan AV, Luber BM, Lisanby SH. The dynamic duo: combining noninvasive brain stimulation with cognitive interventions. Prog Neuropsychopharmacol Biol Psychiatry. 2019;89:347-60.

19 Yavari F, Nitsche MA, Ekhtiari H. transcranial electric stimulation for precision medicine: a spatiomechanistic framework. Front Hum Neurosci. 2017;11:159.

20 Milev RV, Giacobbe P, Kennedy SH, Blumberger DM, Daskalakis ZJ, Downar J, et al. Canadian Network for Mood and Anxiety Treatments (CANMAT) 2016 clinical guidelines for the management of adults with major depressive disorder: section 4. Neurostimulation treatments. Can J Psychiatry. 2016;61:561-75.

21 Thielscher A, Antunes A, Saturnino GB. Field modeling for transcranial magnetic stimulation: a useful tool to understand the physiological effects of TMS? Conf Proc IEEE Eng Med Biol Soc. 2015;2015:222-5.

22 Klooster DC, de Louw AJ, Aldenkamp AP, Besseling RM, Mestrom RM, Carrette S, et al. Technical aspects of neurostimulation: focus on equipment, electric field modeling, and stimulation protocols. Neurosci Biobehav Rev. 2016;65:113-41.

23 Cretaz E, Brunoni AR, Lafer B. Magnetic seizure therapy for unipolar and bipolar depression: a systematic review. Neural Plast. 2015; 2015:521398.

24 Rossi S, Hallett M, Rossini PM, Pascual-Leone A. Safety of TMS Consensus Group. Safety, ethical considerations, and application guidelines for the use of transcranial magnetic stimulation in clinical practice and research. Clin Neurophysiol. 2009;120:2008-39.

25 Bikson M, Grossman P, Thomas C, Zannou AL, Jiang J, Adnan T, et al. Safety of transcranial direct current stimulation: evidence based update 2016. Brain Stimul. 2016;9:641-61.

26 Antal A, Alekseichuk I, Bikson M, Brockmoller J, Brunoni AR, Chen $R$, et al. Low intensity transcranial electric stimulation: safety, ethical, legal regulatory and application guidelines. Clin Neurophysiol. 2017;128:1774-809.

27 Bikson M, Brunoni AR, Charvet LE, Clark VP, Cohen LG, Deng ZD, et al. Rigor and reproducibility in research with transcranial electrical stimulation: an NIMH-sponsored workshop. Brain Stimul. 2018; 11:465-80.

28 Woods AJ, Antal A, Bikson M, Boggio PS, Brunoni AR, Celnik P, et al. A technical guide to tDCS, and related non-invasive brain stimulation tools. Clin Neurophysiol. 2016;127:1031-48.

29 Blumberger DM, Vila-Rodriguez F, Thorpe KE, Feffer K, Noda Y, Giacobbe $P$, et al. Effectiveness of theta burst versus high-frequency repetitive transcranial magnetic stimulation in patients with depression (THREE-D): a randomised non-inferiority trial. Lancet. 2018;391:1683-92.

30 O'Reardon JP, Solvason HB, Janicak PG, Sampson S, Isenberg $\mathrm{KE}$, Nahas Z, et al. Efficacy and safety of transcranial magnetic stimulation in the acute treatment of major depression: a multisite randomized controlled trial. Biol Psychiatry. 2007;62:1208-16.

31 Brunoni AR, Moffa AH, Sampaio-Junior B, Borrione L, Moreno ML, Fernandes RA, et al. Trial of electrical direct-current therapy versus escitalopram for depression. N Engl J Med. 2017;376:2523-33.

32 Pascual-Leone A, Rubio B, Pallardó F, Catalá MD. Rapid-rate transcranial magnetic stimulation of left dorsolateral prefrontal cortex in drug-resistant depression. Lancet. 1996;348:233-7.

33 Fregni F, Boggio PS, Nitsche MA, Marcolin MA, Rigonatti SP, Pascual-Leone A. Treatment of major depression with transcranial direct current stimulation. Bipolar Disord. 2006;8:203-4.

34 Kaiser RH, Andrews-Hanna JR, Wager TD, Pizzagalli DA. Largescale network dysfunction in major depressive disorder: a metaanalysis of resting-state functional connectivity. JAMA Psychiatry. 2015;72:603-11.

35 Padmanabhan JL, Cooke D, Joutsa J, Siddiqi SH, Ferguson M, Darby RR, et al. A human depression circuit derived from focal brain lesions. Biol Psychiatry. 2019;86:749-58.

36 Williams LM. Precision psychiatry: a neural circuit taxonomy for depression and anxiety. Lancet Psychiatry. 2016;3:472-80.

37 Downar J, Blumberger DM, Daskalakis ZJ. The neural crossroads of psychiatric illness: an emerging target for brain stimulation. Trends Cogn Sci. 2016;20:107-20.

38 McTeague LM, Huemer J, Carreon DM, Jiang Y, Eickhoff SB, Etkin A. Identification of common neural circuit disruptions in cognitive control across psychiatric disorders. Am J Psychiatry. 2017;174:676-85. 
39 Baeken C, De Raedt R. Neurobiological mechanisms of repetitive transcranial magnetic stimulation on the underlying neurocircuitry in unipolar depression. Dialogues Clin Neurosci. 2011;13:139-45.

40 Philip NS, Barredo J, van 't Wout-Frank M, Tyrka AR, Price LH, Carpenter LL. Network mechanisms of clinical response to transcranial magnetic stimulation in posttraumatic stress disorder and major depressive disorder. Biol Psychiatry. 2018;83:263-72.

41 Weigand A, Horn A, Caballero R, Cooke D, Stern AP, Taylor SF, et al. Prospective validation that subgenual connectivity predicts antidepressant efficacy of transcranial magnetic stimulation sites. Biol Psychiatry. 2018;84:28-37.

42 Loo C, Katalinic N, Mitchell PB, Greenberg B. Physical treatments for bipolar disorder: a review of electroconvulsive therapy, stereotactic surgery and other brain stimulation techniques. J Affect Disord. 2011;132:1-13.

43 van Buel EM, Patas K, Peters M, Bosker FJ, Eisel UL, Klein HC. Immune and neurotrophin stimulation by electroconvulsive therapy: is some inflammation needed after all? Transl Psychiatry. 2015;5: e609.

44 Brunoni AR, Baeken C, Machado-Vieira R, Gattaz WF, Vanderhasselt MA. BDNF blood levels after electroconvulsive therapy in patients with mood disorders: a systematic review and metaanalysis. World J Biol Psychiatry. 2014;15:411-8.

45 Wilkinson ST, Sanacora G, Bloch MH. Hippocampal volume changes following electroconvulsive therapy: a systematic review and meta-analysis. Biol Psychiatry Cogn Neurosci Neuroimaging. 2017;2:327-35

46 Takamiya A, Chung JK, Liang KC, Graff-Guerrero A, Mimura M, Kishimoto T. Effect of electroconvulsive therapy on hippocampa and amygdala volumes: systematic review and meta-analysis. $\mathrm{Br} \mathrm{J}$ Psychiatry. 2018;212:19-26.

47 Bolwig TG. Neuroimaging and electroconvulsive therapy: a review. J ECT. 2014;30:138-42.

48 Oltedal L, Bartsch H, Sorhaug OJ, Kessler U, Abbott C, Dols A, et al. The Global ECT-MRI Research Collaboration (GEMRIC): establishing a multi-site investigation of the neural mechanisms underlying response to electroconvulsive therapy. Neuroimage Clin. 2017; 14:422-32.

49 Gbyl K, Videbech P. Electroconvulsive therapy increases brain volume in major depression: a systematic review and meta-analysis. Acta Psychiatr Scand. 2018;138:180-95.

50 Hestad KA, Tønseth S, Støen CD, Ueland T, Aukrust P. Raised plasma levels of tumor necrosis factor alpha in patients with depression: normalization during electroconvulsive therapy. J ECT. 2003;19:183-8.

51 Järventausta K, Sorri A, Kampman O, Björkqvist M, Tuohimaa K, Hämäläinen $M$, et al. Changes in interleukin-6 levels during electroconvulsive therapy may reflect the therapeutic response in major depression. Acta Psychiatr Scand. 2017;135:87-92.

52 Roman A, Kreiner G, Nalepa I. Macrophages and depression a misalliance or well-arranged marriage? Pharmacol Rep. 2013;65: 1663-72.

53 Yrondi A, Sporer M, Péran P, Schmitt L, Arbus C, Sauvaget A. Electroconvulsive therapy, depression, the immune system and inflammation: a systematic review. Brain Stimul. 2018;11:29-51.

54 Hallett M. Transcranial magnetic stimulation: a primer. Neuron. 2007;55:187-99.

55 McClintock SM, Reti IM, Carpenter LL, McDonald WM, Dubin M, Taylor SF, et al. consensus recommendations for the clinical application of Repetitive Transcranial Magnetic Stimulation (rTMS) in the treatment of depression. J Clin Psychiatry. 2018;79: $16 \mathrm{cs} 10905$.

56 Lisanby $\mathrm{SH}$, Husain MM, Rosenquist PB, Maixner D, Gutierrez R, Krystal $A$, et al. Daily left prefrontal repetitive transcranial magnetic stimulation in the acute treatment of major depression: clinical predictors of outcome in a multisite, randomized controlled clinical trial. Neuropsychopharmacology. 2009;34:522-34.

57 Berlim MT, Van den Eynde F, Daskalakis ZJ. High-frequency repetitive transcranial magnetic stimulation accelerates and enhances the clinical response to antidepressants in major depression: a meta-analysis of randomized, double-blind, and sham-controlled trials. J Clin Psychiatry. 2013;74:e122-9.

58 Berlim MT, Van den Eynde F, Tovar-Perdomo S, Daskalakis ZJ. Response, remission and drop-out rates following high-frequency
Repetitive Transcranial Magnetic Stimulation (rTMS) for treating major depression: a systematic review and meta-analysis of randomized, double-blind and sham-controlled trials. Psychol Med. 2014;44:225-39.

59 Berlim MT, Van den Eynde F, Daskalakis ZJ. Clinically meaningful efficacy and acceptability of low-frequency Repetitive Transcranial Magnetic Stimulation (rTMS) for treating primary major depression: a meta-analysis of randomized, double-blind and sham-controlled trials. Neuropsychopharmacology. 2013;38:543-51.

60 Brunoni AR, Chaimani A, Moffa AH, Razza LB, Gattaz WF, Daskalakis ZJ, et al. Repetitive Transcranial Magnetic Stimulation for the acute treatment of major depressive episodes: a systematic review with network meta-analysis. JAMA Psychiatry. 2017;74:143-52.

61 Lefaucheur JP, André-Obadia N, Antal A, Ayache SS, Baeken C, Benninger $\mathrm{DH}$, et al. Evidence-based guidelines on the therapeutic use of repetitive transcranial magnetic stimulation (rTMS). Clin Neurophysiol. 2014;125:2150-206.

62 Berlim MT, Turecki G. What is the meaning of treatment resistant/ refractory major depression (TRD)? A systematic review of current randomized trials. Eur Neuropsychopharmacol. 2007;17:696-707.

63 Cohen RB, Boggio PS, Fregni F. Risk factors for relapse after remission with repetitive transcranial magnetic stimulation for the treatment of depression. Depress Anxiety. 2009;26:682-8.

64 Dunner DL, Aaronson ST, Sackeim HA, Janicak PG, Carpenter LL, Boyadjis $\mathrm{T}$, et al. A multisite, naturalistic, observational study of transcranial magnetic stimulation for patients with pharmacoresistant major depressive disorder: durability of benefit over a 1-year follow-up period. J Clin Psychiatry. 2014;75:1394-401.

65 Kedzior KK, Reitz SK, Azorina V, Loo C. Durability of the antidepressant effect of the high-frequency repetitive transcranial magnetic stimulation (rTMS) In the absence of maintenance treatment in major depression: a systematic review and meta-analysis of 16 double-blind, randomized, sham-controlled trials. Depress Anxiety. 2015;32:193-203.

66 Sgro J, Stanton P, Emerson R. Theoretical and practical performance of magnetic stimulators and coils. Electroencephalogr Clin Neurophysiol Suppl. 1991;43:279-83.

67 Counter SA. Neurobiological effects of extensive transcranial electromagnetic stimulation in an animal model. Electroencephalogr Clin Neurophysiol. 1993;89:341-8.

68 Nitsche MA, Cohen LG, Wassermann EM, Priori A, Lang N, Antal A, et al. Transcranial direct current stimulation: state of the art 2008. Brain Stimul. 2008;1:206-23.

69 Brunoni AR, Nitsche MA, Bolognini N, Bikson M, Wagner T, Merabet $\mathrm{L}$, et al. Clinical research with transcranial direct current stimulation (tDCS): challenges and future directions. Brain Stimul. 2012;5: 175-95.

70 Borrione L, Moffa AH, Martin D, Loo CK, Brunoni AR. Transcranial direct current stimulation in the acute depressive episode: a systematic review of current knowledge. J ECT. 2018;34:153-63.

71 Palm U, Hasan A, Strube W, Padberg F. tDCS for the treatment of depression: a comprehensive review. Eur Arch Psychiatry Clin Neurosci. 2016;266:681-94.

72 Brunoni AR, Valiengo L, Baccaro A, Zanão TA, de Oliveira JF, Goulart A, et al. The sertraline vs. electrical current therapy for treating depression clinical study: results from a factorial, randomized, controlled trial. JAMA Psychiatry. 2013;70:383-91.

73 Brunoni AR, Moffa AH, Fregni F, Palm U, Padberg F, Blumberger $\mathrm{DM}$, et al. Transcranial direct current stimulation for acute major depressive episodes: meta-analysis of individual patient data. $\mathrm{Br} \mathrm{J}$ Psychiatry. 2016;208:522-31.

74 Mutz J, Edgcumbe DR, Brunoni AR, Fu CH. Efficacy and acceptability of non-invasive brain stimulation for the treatment of adult unipolar and bipolar depression: a systematic review and metaanalysis of randomised sham-controlled trials. Neurosci Biobehav Rev. 2018;92:291-303.

75 Valiengo L, Benseñor IM, Goulart AC, de Oliveira JF, Zanao TA, Boggio PS, et al. The sertraline versus electrical current therapy for treating depression clinical study (select-TDCS): results of the crossover and follow-up phases. Depress Anxiety. 2013;30:646-53.

76 Aparicio LV, Rosa V, Razza LM, Sampaio-Junior B, Borrione L, Valiengo L, et al. Transcranial direct current stimulation (tDCS) for preventing major depressive disorder relapse: results of a 6-month follow-up. Depress Anxiety. 2019;36:262-8. 
77 Martin DM, Alonzo A, Ho KA, Player M, Mitchell PB, Sachdev P, et al. Continuation transcranial direct current stimulation for the prevention of relapse in major depression. J Affect Disord. 2013; 144:274-8.

78 Alonzo A, Fong J, Ball N, Martin D, Chand N, Loo C. Pilot trial of home-administered transcranial direct current stimulation for the treatment of depression. J Affect Disord. 2019;252:475-83.

79 Alexander ML, Alagapan S, Lugo CE, Mellin JM, Lustenberger C, Rubinow DR, et al. Double-blind, randomized pilot clinical trial targeting alpha oscillations with transcranial alternating current stimulation (tACS) for the treatment of major depressive disorder (MDD). Transl Psychiatry. 2019;9:106.

80 Chan HN, Alonzo A, Martin DM, Player M, Mitchell PB, Sachdev P et al. Treatment of major depressive disorder by transcranial random noise stimulation: case report of a novel treatment. Biol Psychiatry. 2012;72:e9-10.

81 Mirski A, Pachalska M, Moskala M, Orski M, Orska M, Miaskiewicz $M$, et al. Neuromarkers of anxiety and depression in a patient after neuro-ophthalmic surgery of the meningioma-effect of individuallytailored tDCS and neurofeedback. Ann Agric Environ Med. 2015;22: 718-23.

82 Peterchev AV, Rosa MA, Deng ZD, Prudic J, Lisanby SH. Electroconvulsive therapy stimulus parameters: rethinking dosage. J ECT. 2010;26:159-74.

83 Weiner RD, Reti IM. Key updates in the clinical application of electroconvulsive therapy. Int Rev Psychiatry. 2017;29:54-62.

84 UK ECT Review Group. Efficacy and safety of electroconvulsive therapy in depressive disorders: a systematic review and metaanalysis. Lancet. 2003;361:799-808.

85 Kolshus E, Jelovac A, McLoughlin DM. Bitemporal v. high-dose right unilateral electroconvulsive therapy for depression: a systematic review and meta-analysis of randomized controlled trials. Psychol Med. 2017;47:518-30.

86 Dunne RA, McLoughlin DM. Systematic review and meta-analysis of bifrontal electroconvulsive therapy versus bilateral and unilateral electroconvulsive therapy in depression. World J Biol Psychiatry. 2012;13:248-58.

87 Sackeim HA, Haskett RF, Mulsant BH, Thase ME, Mann JJ, Pettinati $\mathrm{HM}$, et al. Continuation pharmacotherapy in the prevention of relapse following electroconvulsive therapy: a randomized controlled trial. JAMA. 2001;285:1299-307.

88 Prudic J, Haskett RF, McCall WV, Isenberg K, Cooper T, Rosenquist $\mathrm{PB}$, et al. Pharmacological strategies in the prevention of relapse after electroconvulsive therapy. J ECT. 2013;29:3-12.

89 Sienaert P. Based on a true story? The portrayal of ECT in international movies and television programs. Brain Stimul. 2016;9:882-91.

90 Ingram A, Saling MM, Schweitzer I. Cognitive side effects of brief pulse electroconvulsive therapy: a review. J ECT. 2008;24:3-9.

91 Andrade C, Arumugham SS, Thirthalli J. Adverse effects of electroconvulsive therapy. Psychiatr Clin North Am. 2016;39:513-30.

92 Deng ZD, Lisanby SH, Peterchev AV. Electric field strength and focality in electroconvulsive therapy and magnetic seizure therapy: a finite element simulation study. J Neural Eng. 2011;8:016007.

93 McClintock SM, Tirmizi O, Chansard M, Husain MM. A systematic review of the neurocognitive effects of magnetic seizure therapy. Int Rev Psychiatry. 2011;23:413-23.

94 Deng Z, Peterchev AV, Krystal AD, Luber B, McClintock SM, Husain $\mathrm{MM}$, et al. Topography of seizures induced by electroconvulsive therapy and magnetic seizure therapy. In: 6th International IEEE/ EMBS Conference on Neural Engineering (NER); 2013; San Diego: USA. p. 577-80.

95 Fitzgerald PB, Hoy KE, Herring SE, Clinton AM, Downey G, Daskalakis ZJ. Pilot study of the clinical and cognitive effects of high-frequency magnetic seizure therapy in major depressive disorder. Depress Anxiety. 2013;30:129-36.

96 Fitzgerald PB, Hoy KE, Elliot D, McQueen S, Wambeek LE, Chen L, et al. A pilot study of the comparative efficacy of $100 \mathrm{~Hz}$ magnetic seizure therapy and electroconvulsive therapy in persistent depression. Depress Anxiety. 2018;35:393-401.

97 Fregni F, Nitsche MA, Loo CK, Brunoni AR, Marangolo P, Leite J, et al. Regulatory considerations for the clinical and research use of transcranial direct current stimulation (tDCS): review and recommendations from an expert panel. Clin Res Regul Aff. 2015;32: 22-35.
98 Insel TR. The NIMH experimental medicine initiative. World Psychiatry. 2015;14:151-3.

99 Li CT, Hsieh JC, Huang $\mathrm{HH}$, Chen MH, Juan $\mathrm{CH}$, Tu PC, et al. Cognition-modulated frontal activity in prediction and augmentation of antidepressant efficacy: a randomized controlled pilot study. Cereb Cortex. 2016;26:202-10.

100 Neacsiu AD, Luber BM, Davis SW, Bernhardt E, Strauman TJ, Lisanby $\mathrm{SH}$. On the concurrent use of self-system therapy and functional magnetic resonance imaging-guided transcranial magnetic stimulation as treatment for depression. J ECT. 2018;34:266-73.

101 Donse L, Padberg F, Sack AT, Rush AJ, Arns M. Simultaneous rTMS and psychotherapy in major depressive disorder: clinical outcomes and predictors from a large naturalistic study. Brain Stimul. 2018;11:337-45.

102 Fritsch B, Reis J, Martinowich K, Schambra HM, Ji Y, Cohen LG, et al. Direct current stimulation promotes BDNF-dependent synaptic plasticity: potential implications for motor learning. Neuron. 2010;66:198-204

103 Reis J, Schambra HM, Cohen LG, Buch ER, Fritsch B, Zarahn E, et al. Noninvasive cortical stimulation enhances motor skill acquisition over multiple days through an effect on consolidation. Proc Natl Acad Sci U S A. 2009;106:1590-5.

104 O'Shea J, Revol P, Cousijn H, Near J, Petitet P, Jacquin-Courtois S, et al. Induced sensorimotor cortex plasticity remediates chronic treatment-resistant visual neglect. Elife. 2017;6.

105 Jackson MP, Rahman A, Lafon B, Kronberg G, Ling D, Parra LC, et al. Animal models of transcranial direct current stimulation: methods and mechanisms. Clin Neurophysiol. 2016;127:3425-54.

106 Bajbouj M, Padberg F. A perfect match: noninvasive brain stimulation and psychotherapy. Eur Arch Psychiatry Clin Neurosci. 2014;264 Suppl 1:S27-33.

107 Martin DM, Teng JZ, Lo TY, Alonzo A, Goh T, lacoviello BM, et al. Clinical pilot study of transcranial direct current stimulation combined with cognitive emotional training for medication resistant depression. J Affect Disord. 2018;232:89-95.

108 Monnart A, Vanderhasselt MA, Schroder E, Campanella S, Fontaine $\mathrm{P}$, Kornreich C. Treatment of resistant depression: a pilot study assessing the efficacy of a tDCS-mindfulness program compared with a tDCS-relaxation program. Front Psychiatry. 2019;10:730.

109 Bajbouj M, Aust S, Spies J, Herrera-Melendez AL, Mayer SV, Peters $M$, et al. PsychotherapyPlus: augmentation of cognitive behavioral therapy (CBT) with prefrontal transcranial direct current stimulation (tDCS) in major depressive disorder--study design and methodology of a multicenter double-blind randomized placebocontrolled trial. Eur Arch Psychiatry Clin Neurosci. 2018;268: 797-808.

110 Brem AK, Almquist JN, Mansfield K, Plessow F, Sella F, Santarnecchi $E$, et al. Modulating fluid intelligence performance through combined cognitive training and brain stimulation. Neuropsychologia. 2018;118:107-14.

111 Nienow TM, MacDonald AW 3rd, Lim KO. TDCS produces incremental gain when combined with working memory training in patients with schizophrenia: a proof of concept pilot study. Schizophr Res. 2016;172:218-9.

112 Martin DM, Mohan A, Alonzo A, Gates N, Gbadeyan O, Meinzer M et al. A pilot double-blind randomized controlled trial of cognitive training combined with transcranial direct current stimulation for amnestic mild cognitive impairment. J Alzheimers Dis. 2019;71: 503-12.

113 Lawrence BJ, Gasson N, Johnson AR, Booth L, Loftus AM. Cognitive training and transcranial direct current stimulation for mild cognitive impairment in Parkinson's disease: a randomized controlled trial. Parkinsons Dis. 2018;2018:4318475.

114 Health Quality Ontario. Psychotherapy for major depressive disorder and generalized anxiety disorder: a health technology assessment. Ont Health Technol Assess Ser. 2017;17:1-167.

115 Bergmann TO, Karabanov A, Hartwigsen G, Thielscher A, Siebner HR. Combining non-invasive transcranial brain stimulation with neuroimaging and electrophysiology: current approaches and future perspectives. Neuroimage. 2016;140:4-19.

116 Thut G, Bergmann TO, Fröhlich F, Soekadar SR, Brittain JS Valero-Cabré A, et al. Guiding transcranial brain stimulation by EEG/MEG to interact with ongoing brain activity and associated functions: a position paper. Clin Neurophysiol. 2017;128:843-57. 
117 Karabanov AN, Saturnino GB, Thielscher A, Siebner HR. Can transcranial electrical stimulation localize brain function? Front Psychol. 2019;10:213.

118 de Lara LI, Windischberger C, Kuehne A, Woletz M, Sieg J, Bestmann S, et al. A novel coil array for combined TMS/fMRI experiments at 3 T. Magn Reson Med2015;74:1492-501.

119 Tik M, Hoffmann A, Sladky R, Tomova L, Hummer A, Navarro de Lara $L$, et al. Towards understanding rTMS mechanism of action: stimulation of the DLPFC causes network-specific increase in functional connectivity. Neuroimage. 2017;162:289-96.

120 Vink JJ, Mandija S, Petrov PI, van den Berg CA, Sommer IE, Neggers SF. A novel concurrent TMS-fMRI method to reveal propagation patterns of prefrontal magnetic brain stimulation. Hum Brain Mapp. 2018;39:4580-92.

121 Ironside M, Browning M, Ansari TL, Harvey CJ, Sekyi-Djan MN Bishop SJ, et al. Effect of prefrontal cortex stimulation on regulation of amygdala response to threat in individuals with trait anxiety: a randomized clinical trial. JAMA Psychiatry. 2019;76:71-8.

122 Mir-Moghtadaei A, Caballero R, Fried P, Fox MD, Lee K, Giacobbe $\mathrm{P}$, et al. Concordance between beamF3 and MRI-neuronavigated target sites for repetitive transcranial magnetic stimulation of the left dorsolateral prefrontal cortex. Brain Stimul. 2015;8:965-73.

123 Seibt O, Brunoni AR, Huang Y, Bikson M. The pursuit of DLPFC: non-neuronavigated methods to target the left dorsolateral prefrontal cortex with symmetric bicephalic transcranial direct current stimulation (tDCS). Brain Stimul. 2015;8:590-602.

124 Fox MD, Buckner RL, White MP, Greicius MD, Pascual-Leone A. Efficacy of transcranial magnetic stimulation targets for depression is related to intrinsic functional connectivity with the subgenual cingulate. Biol Psychiatry. 2012;72:595-603.

125 Opitz A, Yeagle E, Thielscher A, Schroeder C, Mehta AD, Milham MP. On the importance of precise electrode placement for targeted transcranial electric stimulation. Neuroimage. 2018;181:560-7.

126 Ruffini G, Fox MD, Ripolles O, Miranda PC, Pascual-Leone A. Optimization of multifocal transcranial current stimulation for weighted cortical pattern targeting from realistic modeling of electric fields. Neuroimage. 2014;89:216-25.

127 Beynel L, Appelbaum LG, Luber B, Crowell CA, Hilbig SA, Lim W, et al. Effects of online repetitive transcranial magnetic stimulation (rTMS) on cognitive processing: a meta-analysis and recommendations for future studies. Neurosci Biobehav Rev. 2019;107:47-58.

128 Huang YZ, Lu MK, Antal A, Classen J, Nitsche M, Ziemann U, et al. Plasticity induced by non-invasive transcranial brain stimulation: a position paper. Clin Neurophysiol. 2017;128:2318-29.

129 Ziemann U, Reis J, Schwenkreis P, Rosanova M, Strafella A, Badawy R, et al. TMS and drugs revisited 2014. Clin Neurophysiol. 2015;126:1847-68.

130 Truong D, Adair D, Bikson M. Computer-based models of tDCS and tACS. In: Brunoni AR, Nitsche M, Loo CK, editors. Transcranial direct current stimulation in neuropsychiatric disorders: clinical principles and management. New York: Springer; 2016. p. 47-66.

131 Brunoni AR, Moffa AH, Fregni F, Palm U, Padberg F, Blumberger DM, et al. Transcranial direct current stimulation for acute major depressive episodes: meta-analysis of individual patient data. $\mathrm{Br} \mathrm{J}$ Psychiatry. 2016;208:522-31.

132 Brakemeier EL, Wilbertz G, Rodax S, Danker-Hopfe H, Zinka B, Zwanzger $P$, et al. Patterns of response to repetitive transcranial magnetic stimulation (rTMS) in major depression: replication study in drug-free patients. J Affect Disord. 2008;108:59-70.

133 Haq AU, Sitzmann AF, Goldman ML, Maixner DF, Mickey BJ. Response of depression to electroconvulsive therapy: a meta-analysis of clinical predictors. J Clin Psychiatry. 2015;76:1374-84.

134 Feffer K, Lee HH, Mansouri F, Giacobbe P, Vila-Rodriguez F, Kennedy SH, et al. Early symptom improvement at 10 sessions as a predictor of rTMS treatment outcome in major depression. Brain Stimul. 2018;11:181-9.

135 Birkenhager TK, Roos J, Kamperman AM. Improvement after two sessions of electroconvulsive therapy predicts final remission in inpatients with major depression. Acta Psychiatr Scand. 2019;140: 189-95.

136 Loo CK, Husain MM, McDonald WM, Aaronson S, O'Reardon JP, Alonzo A, et al. International randomized-controlled trial of transcranial direct current stimulation in depression. Brain Stimul. 2018;11:125-33.
137 Batsikadze G, Moliadze V, Paulus W, Kuo MF, Nitsche MA. Partially non-linear stimulation intensity-dependent effects of direct current stimulation on motor cortex excitability in humans. J Physiol. 2013;591:1987-2000.

138 Alonzo A, Chan G, Martin D, Mitchell PB, Loo C. Transcranial direct current stimulation (tDCS) for depression: analysis of response using a three-factor structure of the Montgomery-Åsberg depression rating scale. J Affect Disord. 2013;150:91-5.

139 Cocchi L, Zalesky A. Personalized transcranial magnetic stimulation in psychiatry. Biol Psychiatry Cogn Neurosci Neuroimaging. 2018; 3:731-41.

140 Drysdale AT, Grosenick L, Downar J, Dunlop K, Mansouri F, Meng $\mathrm{Y}$, et al. Resting-state connectivity biomarkers define neurophysiological subtypes of depression. Nat Med. 2017;23:28-38.

141 Kedzior KK, Azorina V, Reitz SK. More female patients and fewer stimuli per session are associated with the short-term antidepressant properties of repetitive transcranial magnetic stimulation (rTMS): a meta-analysis of 54 sham-controlled studies published between 1997-2013. Neuropsychiatr Dis Treat. 2014;10:727-56.

142 Poulet E, Galvao F, Haffen E, Szekely D, Brault C, Haesebaert F, et al. Effects of smoking status and MADRS retardation factor on response to low frequency repetitive transcranial magnetic stimulation for depression. Eur Psychiatry. 2016;38:40-4.

143 Okazaki M, Tominaga K, Higuchi H, Utagawa I, Nakamura E, Noguchi M, et al. Predictors of response to electroconvulsive therapy obtained using the three-factor structure of the Montgomery and Asberg depression rating scale for treatment-resistant depressed patients. J ECT. 2010;26:87-90.

144 O'Reardon JP, Solvason HB, Janicak PG, Sampson S, Isenberg $\mathrm{KE}$, Nahas Z, et al. Reply regarding "efficacy and safety of transcranial magnetic stimulation in the acute treatment of major depression: a multisite randomized controlled trial.”. Biol Psychiatry. 2010;67:e15-7.

145 Chekroud AM, Zotti RJ, Shehzad Z, Gueorguieva R, Johnson MK, Trivedi $\mathrm{MH}$, et al. Cross-trial prediction of treatment outcome in depression: a machine learning approach. Lancet Psychiatry. 2016;3:243-50.

146 Chekroud AM, Gueorguieva R, Krumholz HM, Trivedi MH, Krystal $\mathrm{JH}$, McCarthy G. Reevaluating the efficacy and predictability of antidepressant treatments: a symptom clustering approach. JAMA Psychiatry. 2017;74:370-8.

147 Bzdok D, Altman N, Krzywinski M. Statistics versus machine learning. Nat Methods. 2018;15:233-4.

148 Passos IC, Mwangi B, Kapczinski F. Big data analytics and machine learning: 2015 and beyond. Lancet Psychiatry. 2016;3:13-5.

149 Passos IC, Ballester PL, Barros RC, Librenza-Garcia D, Mwangi B, Birmaher B, et al. Machine learning and big data analytics in bipolar disorder: A position paper from the International Society for Bipolar Disorders Big Data Task Force. Bipolar Disord. 2019;21:582-94.

150 Rashed EA, Sakai T, Gomez-Tames J, Hirata A. Brain Al: deep learning for brain stimulation. IEEE Pulse. 2019;10:3-5.

151 Davis J, Maes M, Andreazza A, McGrath JJ, Tye SJ, Berk M. Towards a classification of biomarkers of neuropsychiatric disease: from encompass to compass. Mol Psychiatry. 2015;20:152-3.

152 Boes AD, Uitermarkt BD, Albazron FM, Lan MJ, Liston C, PascualLeone $A$, et al. Rostral anterior cingulate cortex is a structural correlate of repetitive TMS treatment response in depression. Brain Stimul. 2018;11:575-81.

153 Furtado CP, Hoy KE, Maller JJ, Savage G, Daskalakis ZJ, Fitzgerald PB. An investigation of medial temporal lobe changes and cognition following antidepressant response: a prospective rTMS study. Brain Stimul. 2013;6:346-54.

154 Lan MJ, Chhetry BT, Liston C, Mann JJ, Dubin M. Transcranial magnetic stimulation of left dorsolateral prefrontal cortex induces brain morphological changes in regions associated with a treatment resistant major depressive episode: an exploratory analysis. Brain Stimul. 2016;9:577-83.

155 Hayasaka S, Nakamura M, Noda Y, Izuno T, Saeki T, Iwanari H, et al. Lateralized hippocampal volume increase following high-frequency left prefrontal repetitive transcranial magnetic stimulation in patients with major depression. Psychiatry Clin Neurosci. 2017;71: 747-58.

156 Downar J, Geraci J, Salomons TV, Dunlop K, Wheeler S, McAndrews MP, et al. Anhedonia and reward-circuit connectivity 
distinguish nonresponders from responders to dorsomedial prefrontal repetitive transcranial magnetic stimulation in major depression. Biol Psychiatry. 2014;76:176-85.

157 Iwabuchi SJ, Auer DP, Lankappa ST, Palaniyappan L. Baseline effective connectivity predicts response to repetitive transcranial magnetic stimulation in patients with treatment-resistant depression. Eur Neuropsychopharmacol. 2019;29:681-90.

158 Avissar M, Powell F, llieva I, Respino M, Gunning FM, Liston C, et al. Functional connectivity of the left DLPFC to striatum predicts treatment response of depression to TMS. Brain Stimul. 2017;10: 919-25.

159 Dinga R, Schmaal L, Pennix BW, van Tol MJ, Veltman DJ, van Velzen L, et al. Evaluating the evidence for biotypes of depression: methodological replication and extension of Drysdale et al. (2017). Neuroimage Clin. 2019;22:101796.

160 Bulubas L, Padberg F, Bueno PV, Duran F, Busatto G, Amaro E Jr, et al. Antidepressant effects of tDCS are associated with prefrontal gray matter volumes at baseline: Evidence from the ELECT-TDCS trial. Brain Stimul. 2019;12:1197-204.

161 Dunlop K, Talishinsky A, Liston C. Intrinsic brain network biomarkers of antidepressant response: a review. Curr Psychiatry Rep. 2019;21:87.

162 Doumas M, Smolders C, Brunfaut E, Bouckaert F, Krampe RT. Dual task performance of working memory and postural control in major depressive disorder. Neuropsychology. 2012;26:110-8.

163 Reppermund S, Zihl J, Lucae S, Horstmann S, Kloiber S, Holsboer $\mathrm{F}$, et al. Persistent cognitive impairment in depression: the role of psychopathology and altered hypothalamic-pituitary-adrenocortical (HPA) system regulation. Biol Psychiatry. 2007;62:400-6.

164 Snyder HR. Major depressive disorder is associated with broad impairments on neuropsychological measures of executive function: a meta-analysis and review. Psychol Bull. 2013;139:81-132.

165 Gotlib IH, Joormann J. Cognition and depression: current status and future directions. Annu Rev Clin Psychol. 2010;6:285-312.

166 Gyurak A, Patenaude B, Korgaonkar MS, Grieve SM, Williams LM, Etkin A. Frontoparietal activation during response inhibition predicts remission to antidepressants in patients with major depression. Biol Psychiatry. 2016;79:274-81.

167 Rossi S, Hallett M, Rossini PM, Pascual-Leone A; Safety of TMS Consensus Group. Safety, ethical considerations, and application guidelines for the use of transcranial magnetic stimulation in clinical practice and research. Clin Neurophysiol. 2009;120:2008-39.

168 Brunoni AR, Zanao TA, Ferrucci R, Priori A, Valiengo L, de Oliveira $\mathrm{JF}$, et al. Bifrontal tDCS prevents implicit learning acquisition in antidepressant-free patients with major depressive disorder. Prog Neuropsychopharmacol Biol Psychiatry. 2013;43:146-50.

169 Sellers KK, Mellin JM, Lustenberger CM, Boyle MR, Lee WH, Peterchev AV, et al. Transcranial direct current stimulation (tDCS) of frontal cortex decreases performance on the WAIS-IV intelligence test. Behav Brain Res. 2015;290:32-44.

170 limori T, Nakajima S, Miyazaki T, Tarumi R, Ogyu K, Wada M, et al. Effectiveness of the prefrontal repetitive transcranial magnetic stimulation on cognitive profiles in depression, schizophrenia, and Alzheimer's disease: a systematic review. Prog Neuropsychopharmacol Biol Psychiatry. 2019;88:31-40.

171 Martin DM, Moffa A, Nikolin S, Bennabi D, Brunoni AR, Flannery W, et al. Cognitive effects of transcranial direct current stimulation treatment in patients with major depressive disorder: an individual patient data meta-analysis of randomised, sham-controlled trials. Neurosci Biobehav Rev. 2018;90:137-45.

172 Brunoni AR, Vanderhasselt MA. Working memory improvement with non-invasive brain stimulation of the dorsolateral prefrontal cortex: a systematic review and meta-analysis. Brain Cogn. 2014; $86: 1-9$.

173 Martin DM, McClintock SM, Forster JJ, Lo TY, Loo CK. Cognitive enhancing effects of rTMS administered to the prefrontal cortex in patients with depression: A systematic review and meta-analysis of individual task effects. Depress Anxiety. 2017;34:1029-39.

174 Tortella G, Selingardi PM, Moreno ML, Veronezi BP, Brunoni AR. Does non-invasive brain stimulation improve cognition in major depressive disorder? A systematic review. CNS Neurol Disord Drug Targets. 2014;13:1759-69.

175 Brunoni AR, Tortella G, Benseñor IM, Lotufo PA, Carvalho AF, Fregni $F$. Cognitive effects of transcranial direct current stimulation in depression: results from the SELECT-TDCS trial and insights for further clinical trials. J Affect Disord. 2016;202:46-52.

176 Martin DM, McClintock SM, Aaronson ST, Alonzo A, Husain MM, Lisanby $\mathrm{SH}$, et al. Pre-treatment attentional processing speed and antidepressant response to transcranial direct current stimulation: results from an international randomized controlled trial. Brain Stimul. 2018;11:1282-90.

177 Vanderhasselt M, De Raedt R, Leyman L, Baeken C. Immediate effects of repetitive transcranial magnetic stimulation on attentional processes are associated with antidepressant treatment outcome. Brain Stimul. 2008;1:249.

178 Beaudreau SA, Rideaux T, O'Hara R, Arean P. Does cognition predict treatment response and remission in psychotherapy for latelife depression? Am J Geriatr Psychiatry. 2015;23:215-9.

179 Hellström LC, Eplov LF, Nordentoft M, Østergaard SD, Bech P. The Diagnostic Apathia Scale predicts the ability to return to work following depression or anxiety. Acta Neuropsychiatr. 2014;26:364-71.

180 Harty S, Sella F, Kadosh RC. Transcranial electrical stimulation and behavioral change: the intermediary influence of the brain. Front Hum Neurosci. 2017;11:112.

181 Roick H, von Giesen HJ, Benecke R. On the origin of the postexcitatory inhibition seen after transcranial magnetic brain stimulation in awake human subjects. Exp Brain Res. 1993;94:489-98.

182 Rossini PM, Burke D, Chen R, Cohen LG, Daskalakis Z, Di lorio R, et al. Non-invasive electrical and magnetic stimulation of the brain, spinal cord, roots and peripheral nerves: basic principles and procedures for routine clinical and research application. An updated report from an I.F.C.N. Committee. Clin Neurophysiol. 2015;126: 1071-107.

183 Oliveira-Maia AJ, Press D, Pascual-Leone A. Modulation of motor cortex excitability predicts antidepressant response to prefrontal cortex repetitive transcranial magnetic stimulation. Brain Stimul. 2017; 10:787-94.

184 Bajbouj M, Lang UE, Niehaus L, Hellen FE, Heuser I, Neu P. Effects of right unilateral electroconvulsive therapy on motor cortical excitability in depressive patients. J Psychiatr Res. 2006;40:322-7.

185 Arns M, Drinkenburg WH, Fitzgerald PB, Kenemans JL. Neurophysiological predictors of non-response to rTMS in depression. Brain Stimul. 2012;5:569-76.

186 Shalbaf R, Brenner C, Pang C, Blumberger DM, Downar J, Daskalakis ZJ, et al. Non-linear entropy analysis in EEG to predict treatment response to repetitive transcranial magnetic stimulation in depression. Front Pharmacol. 2018;9:1188.

187 Hui J, Tremblay S, Daskalakis ZJ. The Current and future potential of transcranial magnetic stimulation with electroencephalography in psychiatry. Clin Pharmacol Ther. 2019;106:734-46.

188 Sun Y, Blumberger DM, Mulsant BH, Rajji TK, Fitzgerald PB, Barr MS, et al. Magnetic seizure therapy reduces suicidal ideation and produces neuroplasticity in treatment-resistant depression. Transl Psychiatry. 2018;8:253.

189 Voineskos D, Blumberger DM, Zomorrodi R, Rogasch NC, Farzan F, Foussias G, et al. Altered transcranial magnetic stimulationelectroencephalographic markers of inhibition and excitation in the dorsolateral prefrontal cortex in major depressive disorder. Biol Psychiatry. 2019;85:477-86.

190 Sun Y, Farzan F, Mulsant BH, Rajji TK, Fitzgerald PB, Barr MS, et al. Indicators for remission of suicidal ideation following magnetic seizure therapy in patients with treatment-resistant depression. JAMA Psychiatry. 2016;73:337-45.

191 Brunoni AR, Machado-Vieira R, Zarate CA, Vieira EL, Valiengo L, Bensenor IM, et al. Assessment of non-BDNF neurotrophins and GDNF levels after depression treatment with sertraline and transcranial direct current stimulation in a factorial, randomized, shamcontrolled trial (SELECT-TDCS): An exploratory analysis. Prog Neuropsychopharmacol Biol Psychiatry. 2015;56:91-6.

192 Brunoni AR, Machado-Vieira R, Zarate CA Jr, Vieira EL, Vanderhasselt MA, Nitsche MA, et al. BDNF plasma levels after antidepressant treatment with sertraline and transcranial direct current stimulation: Results from a factorial, randomized, sham-controlled trial. Eur Neuropsychopharmacol. 2014;24:1144-51.

193 Brunoni AR, Padberg F, Vieira EL, Teixeira AL, Carvalho AF, Lotufo $\mathrm{PA}$, et al. Plasma biomarkers in a placebo-controlled trial comparing tDCS and escitalopram efficacy in major depression. Prog Neuropsychopharmacol Biol Psychiatry. 2018;86:211-7. 
194 Brunoni AR, Kemp AH, Shiozawa P, Cordeiro Q, Valiengo LC, Goulart AC, et al. Impact of 5-HTTLPR and BDNF polymorphisms on response to sertraline versus transcranial direct current stimulation: implications for the serotonergic system. Eur Neuropsychopharmacol. 2013;23:1530-40.

195 Noda Y, Silverstein WK, Barr MS, Vila-Rodriguez F, Downar J, Rajji TK, et al. Neurobiological mechanisms of repetitive transcranial magnetic stimulation of the dorsolateral prefrontal cortex in depression: a systematic review. Psychol Med. 2015;45:3411-32.

196 Silverstein WK, Noda Y, Barr MS, Vila-Rodriguez F, Rajji TK, Fitzgerald PB, et al. Neurobiological predictors of response to dorsolateral prefrontal cortex repetitive transcranial magnetic stimulation in depression: a systematic review. Depress Anxiety. 2015;32: 871-91.

197 Brunoni AR, Kemp AH, Dantas EM, Goulart AC, Nunes MA, Boggio $P S$, et al. Heart rate variability is a trait marker of major depressive disorder: evidence from the sertraline vs. electric current therapy to treat depression clinical study. Int $\mathrm{J}$ Neuropsychopharmacol. 2013;16:1937-49.

198 Brunoni AR, Baeken C, Machado-Vieira R, Gattaz WF, Vanderhasselt MA. BDNF blood levels after non-invasive brain stimulation interventions in major depressive disorder: a systematic review and meta-analysis. World J Biol Psychiatry. 2015;16:114-22.

199 Fidalgo TM, Morales-Quezada JL, Muzy GS, Chiavetta NM, Mendonca ME, Santana MV, et al. Biological markers in noninvasive brain stimulation trials in major depressive disorder: a systematic review. J ECT. 2014;30:47-61.

200 Border R, Johnson EC, Evans LM, Smolen A, Berley N, Sullivan PF, et al. No support for historical candidate gene or candidate gene-byinteraction hypotheses for major depression across multiple large samples. Am J Psychiatry. 2019;176:376-87.

201 Carvalho AF, Köhler CA, Brunoni AR, Miskowiak KW, Herrmann N Lanctôt $\mathrm{KL}$, et al. Bias in peripheral depression biomarkers. Psychother Psychosom. 2016;85:81-90.

202 Carvalho AF, Kohler CA, Fernandes BS, Quevedo J, Miskowiak $\mathrm{KW}$, Brunoni AR, et al. Bias in emerging biomarkers for bipolar disorder. Psychol Med. 2016;46:2287-97.

203 George MS. Brain Stimulation's expanding impact -- Now immediately free to download by anyone, anywhere and at anytime. Brain Stimul. 2020;13:277-9.

204 Passos IC, Mwangi B. Machine learning-guided intervention trials to predict treatment response at an individual patient level: an important second step following randomized clinical trials. Mol Psychiatry. 2018 Sep 21. doi: 10.1038/s41380-018-0250-y. [Epub ahead of print]

205 Brunoni AR, Fregni F, Pagano RL. Translational research in transcranial direct current stimulation (tDCS): a systematic review of studies in animals. Rev Neurosci. 2011;22:471-81.

206 Lorenz R, Simmons LE, Monti RP, Arthur JL, Limal S, Laakso I, et al. Efficiently searching through large tACS parameter spaces using closed-loop Bayesian optimization. Brain Stimul. 2019;12:1484-9. 